\title{
piRNA-mediated gene regulation and adaptation to sex-specific transposon expression in $D$. melanogaster male germline
}

\author{
Peiwei Chen ${ }^{1 * \&}$, Alexei A. Kotov², Baira K. Godneeva ${ }^{1}$, Sergei S. Bazylev², \\ Ludmila V. Olenina ${ }^{2}$ and Alexei A. Aravin ${ }^{18}$
}

${ }^{1}$ California Institute of Technology,

Division of Biology and Biological Engineering

Pasadena, CA 91125, USA

${ }^{2}$ Institute of Molecular Genetics,

Russian Academy of Sciences,

Moscow 123182, Russia

* These authors contributed equally

\&To whom correspondence should be addressed:

Alexei Aravin aaa@caltech.edu

Peiwei Chen peiweitc@gmail.com 


\section{SUMMARY}

Small non-coding piRNAs act as sequence-specific guides to repress complementary targets in Metazoa. Prior studies in Drosophila ovaries have demonstrated the function of piRNA pathway in transposon silencing and therefore genome defense. However, the ability of piRNA program to respond to different transposon landscape and the role of piRNAs in regulating host gene expression remain poorly understood. Here, we comprehensively analyzed piRNA expression and defined the repertoire of their targets in Drosophila melanogaster testes. Comparison of piRNA programs between sexes revealed sexual dimorphism in piRNA programs that parallel sex-specific transposon expression. Using a novel bioinformatic pipeline, we identified new piRNA clusters and established complex satellites as dual-strand piRNA clusters. While sharing most piRNA clusters, two sexes employ them differentially to combat sex-specific transposon landscape. We found several host genes targeted by piRNAs in testis, including CG12717/pita, a SUMO protease gene. piRNAs encoded on $\mathrm{Y}$ chromosome silence pita, but not its paralog, to exert sex- and paralog-specific gene regulation. Interestingly, pita is targeted by endogenous siRNAs in a sibling species, Drosophila mauritiana, suggesting distinct but related silencing strategies invented in recent evolution to regulate a conserved protein-encoding gene. 


\section{INTRODUCTION}

PIWl-interacting (pi)RNA is a class of small non-coding RNAs named after their interaction with PIWI-clade Argounate proteins. piRNAs guide PIWI proteins to complementary RNAs, thereby specifying the target of PIWI silencing. Unlike miRNAs and siRNAs that are ubiquitously expressed, the expression of piRNAs is restricted to gonads in many animals. As a result, perturbation of the piRNA program often compromises reproductive functions with no obvious defects in soma. Drosophila melanogaster is one of the most used model organisms to study piRNA biogenesis and function. In fact, piRNAs were first described in fly testes (Aravin et al., 2001; Vagin et al., 2006). However, most subsequent studies were performed using ovaries as a model system. Work on female gonads has shown that most piRNAs have homology to transposable elements (TEs), suggesting TEs as major targets of piRNAs (Brennecke et al., 2007). Studies on fly ovaries also identified large intergenic regions dubbed piRNA clusters that harbor nested TE fragments, which act as genomic source loci of piRNAs. A peri-centromeric region on chr2R called $42 A B$ was found to be the most active piRNA cluster in ovaries. It remains largely unexplored to what extent these findings from ovaries are applicable to the male counterpart. To date, we still know very little about how sexually dimorphic the Drosophila piRNA program is, besides that there is a single locus on $\mathrm{Y}$ chromosome called Suppressor of Stellate (Su(Ste)) that produces piRNAs only in males.

Importantly, Drosophila as an animal model offers unique value to studying sexual dimorphism of the piRNA program in general. In zebrafish, piRNA pathway mutants are always phenotypically males (Houwing et al., 2007, 2008; Kamminga et al., 2010), rendering it nearly impossible to probe the impact of piRNA loss in females. In mice, an intact piRNA program is only required for male fertility, while murine females are insensitive to piRNA loss (Carmell et al., 2007; Deng and Lin, 2002; Kuramochi-Miyagawa et al., 2004). Contrary to fish and mouse, fly fertility is dependent on a functional piRNA pathway in both sexes (Aravin et al., 2001; Brennecke et al., 2007; Lin and Spradling, 1997; Vagin et al., 2006). Therefore, Drosophila provides an unparalleled opportunity to study whether, and if so how, the piRNA program can be modified in each sex to safeguard reproductive functions.

In this study, we comprehensively analyzed the piRNA profile in Drosophila melanogaster testis and compared it to the female counterpart. Besides TEs, we found complex satellites as another class of selfish genetic elements targeted by the piRNA pathway in gonads of both sexes. Our analysis showed that TE-silencing piRNA program is sexually dimorphic, and it shows evidence of adaptation to sex-specific TE landscape. To understand the genomic origins of 
differentially produced piRNAs, we sought to de novo define genome-wide piRNA clusters in testis. However, we noticed that the standard pipeline used for ovary piRNAs failed to detect known piRNA clusters in testis, so we developed a new bioinformatic algorithm to tackle this problem. Using the new algorithm, we were able to identify novel piRNA clusters and to quantify their activities more accurately in both sexes. Notably, piRNA source loci are employed differentially in males and females, and the sex bias of piRNA cluster expression appears to match that of their TE contents. We also found two loci producing piRNAs with the potential to repress host proteinencoding genes, including a newly identified locus on $\mathrm{Y}$ that produces piRNAs against CG12717/pita. Expression of pita, but not its paralog veloren, is de-repressed in multiple piRNA pathway mutants, indicating that piRNAs silence its expression and can distinguish paralogs with sequence similarities. Finally, we explored the evolutionary history of pita and found it to be a young gene conserved in the melanogaster subgroup. Intriguingly, pita is targeted by another class of small non-coding RNAs, endogenous siRNAs, in the sibling species Drosophila mauritiana, suggesting distinct small RNA-based silencing strategies invented in recent evolution to regulate a young yet conserved gene.

\section{RESULTS}

\section{Drosophila piRNA program is sexually dimorphic}

To characterize the piRNA profile in male gonads, we sequenced 18-30nt small RNAs from testes and compared them with published ovary small RNA datasets (EIMaghraby et al., 2019). Mapping and annotation of small RNA reads using the pipeline shown in Figure S1 revealed large differences in the expression of major classes of small RNAs between testes and ovaries. In agreement with previous findings (Czech et al., 2008), TE-mapping 23-29nt piRNAs are the most abundant class of small RNAs in ovaries, while 21-23nt microRNAs constitute a minor fraction and an even smaller one for $21 \mathrm{nt}$ endogenous (endo-) siRNAs (Figure 1A). In contrast, miRNAs constitute a larger fraction in testes, so do endo-siRNAs that map to protein-encoding genes, consistent with a previous report (Wen et al., 2015). To define the piRNA population, we eliminated reads mapping to other types of non-coding RNA (rRNA, miRNA, snRNA, snoRNA and tRNA) from 23-29nt small RNAs. Remaining reads show a strong bias for $U$ at the first nucleotide ("1U bias": 70.9\%), the feature of bona fide piRNAs (Figure 1B). The piRNA-to-miRNA ratio is distinct between sexes: $\sim 10$ in ovary and $\sim 2$ in testis. In both sexes, piRNAs mapping to TEs take up the largest fraction of total piRNAs. However, whereas $66 \%$ of piRNAs mapped to TEs in ovaries, only $40 \%$ mapped to TEs in testes (Figure 1B). Meanwhile, larger fractions of total 
piRNAs mapped to protein-encoding genes (including introns) and intergenic regions in testes (24.6\% and $30.0 \%$, respectively) than ovaries (19.6\% and $10.7 \%$, respectively). These results suggest that distinct piRNA programs operate in male and female gonads.

Testis piRNAs also map to several known complex satellites: HETRP/TAS (a subtelomeric satellite repeat), Responder (Rsp) and $S A R$ (related to 1.688 repeat family) (Figure 1C; Figure S2A). Complex satellite-mapping small RNAs in testis exhibit $1 \mathrm{U}$ bias and size distribution that peaks around 24-26nt, consistent with their piRNA identities. Both strands of complex satellites produce piRNAs, and their production depends on Rhi (see accompanying manuscript), a protein that marks dual-strand piRNA clusters and is required for their expression (Klattenhoff et al., 2009; Mohn et al., 2014; Zhang et al., 2014). Similarly, ovary small RNAs also map to complex satellites and show features of bone fide piRNAs, including $1 \mathrm{U}$ bias, size distribution that peaks around 24-26nt, small RNA production from both strands and dependency on Rhino. Moreover, piRNAs from complex satellites show ping-pong signature, an enrichment for 10nt overlap between the 5' ends of complementary piRNA pairs, except for Rsp in testis (Figure 1C; Figure S2B). These results show that complex satellites are sources of piRNAs in both sexes, pointing to a possible role of piRNAs in regulating satellite DNA and associated heterochromatin in the gonad.

We next analyzed piRNAs targeting different TE families. Comparison of small RNA profiles in testis and ovary showed that piRNAs targeting different TEs are expressed at different levels in two sexes (Figure 2A). Top 3 TEs targeted by piRNA are all different in testis and ovary, and, among top 10, only 3 are shared between sexes (Figure 2B). The most differentially targeted TEs are two telomere-associated TEs, HeT-A and TAHRE, which ovary makes 106 and 74 times more antisense piRNAs, respectively, than testis. In contrast, several elements such as baggins1, invader 3 and copia are targeted by more piRNAs in testis. piRNAs targeting all but one (copia) TE families show stronger ping-pong signature in ovary, as measured by ping-pong z-score (Figure 2A). In conclusion, different TE families are targeted by piRNAs differentially in two sexes.

\section{Distinct piRNA programs in two sexes parallel sex-specific TE expression}

To explore if sex differences in TE-targeting piRNA programs are accompanied by differential expression of TEs themselves, we set out to compare expression levels of different TE families in two sexes. Since piRNA pathway efficiently represses TEs, their expression in wild-type animals does not reflect their full expression potentials that can be achieved when piRNA 
silencing is removed. Hence, we analyzed TE expression in testes and ovaries of rhi mutants that lose piRNA production from dual-strand clusters in both sexes (see accompanying manuscript) and controls.

Profiling TE expression in two sexes by polyA-selected (polyA+) RNA-seq demonstrated clear sexual dimorphism. Overall, TE expression in piRNA pathway mutant testes and ovaries is weakly correlated (Spearman's $\rho$ : 0.26; Figure S3A). Among the 10 most expressed TE families in two sexes, only 5 overlap, though the same element, copia, has the highest expression in both ovary and testis (Figure $2 \mathrm{C}$ ). There are more TE families expressed above each of the three expression cutoffs (1000, 100 and 25 RPM) in ovaries than testes (Figure S3A). The most ovarybiased TEs include Blood, max, Burdock and two telomere-associated TEs, HeT-A and TART (Figure 2E). Only a few TE families are expressed higher in testis than ovary (Figure 2E; Figure $\mathrm{S} 3 \mathrm{~A}$ ). In this group, doc2 shows the highest expression in testis (87 RPM, 8.5-fold higher than ovary). Several elements are expressed at lower levels but have stronger biases for expression in testis: expression of Tom could only be detected in testis but not in ovary, and Transib2 is expressed 28-fold higher in testis than ovary. Overall, the majority of TE families demonstrate strong differences in their expression between sexes.

To quantify the effect of piRNA pathway in suppressing TEs in two sexes, we calculated levels of TE de-repression upon disruption of piRNA pathway. Few TE families remained unaffected by rhi mutation, often accompanied by unperturbed antisense piRNA production (e.g., gypsy, gypsy10 and tabor). There are 9 TE families up-regulated more than 100-fold in ovary. In contrast, no TE is up-regulated that strongly in testis (Figure 2D). Overall, the vast majority of TEs show stronger de-repression in ovaries, with gypsy12 (389-fold), Burdock (317-fold), HeT-A (239fold) and TART (80-fold) being the most prominent examples, as all of them exhibited no or mild de-repression (<4-fold) in testes (Figure 2E). We found only 6 TEs that show stronger (at least 4fold) de-repression in testis than ovary (Transib2, BS2, baggins1, Dm297, invader3, invader6). Altogether, our results show that piRNAs regulate the expression of different TE families to distinct extents in two sexes, with many TEs silenced more in ovary and only a few silenced more in testis.

To explore the link between TE expression and piRNA programs in two sexes, we identified a set of 36 TE families repressed by piRNA pathway in at least one sex (see methods). For these TE families, there is a positive correlation between sex bias of piRNA production and sex bias of TE de-repression (Spearman's $\rho: 0.53, P<0.001$; Figure $3 A$ ). For example, disruption of piRNA pathway by rhi mutations dramatically increases expression of three telomereassociated TEs (HeT-A, TAHRE and TART) in ovaries, where there are abundant piRNAs 
targeting these elements. On the contrary, much fewer piRNAs target these telomeric TEs in testes and expression of these TEs remained very low in rhi mutant males (Figure 3B; Figure 2A and $2 \mathrm{E}$ ). This result indicates that telomeric TEs have a strong, intrinsic bias in their expression towards the female germline, and that piRNA pathway appears to have adapted to this bias generating respective antisense piRNAs in female, but not male, gonads. In contrast to ovarybiased TEs like telomeric elements, testis-biased TEs such as Transib2 and baggins1 are targeted by more antisense piRNAs in testis than ovary (Figure 3B; Figure 2A and 2E). Some TEs, such as copia, mdg3 and l-element are strongly repressed by piRNAs in both sexes. For such elements, the sex bias in piRNA production does not always match that of TE repression (Figure 3A). Taken together, these findings suggest that, for most TEs, piRNA programs in males and females have adapted to differential TE activities between sexes.

To further explore whether differential expression of piRNAs between sexes has functional consequences, we studied Burdock, an LTR retro-transposon targeted by 53 times more piRNAs in ovary (3,756 RPM) than testis (70 RPM) (Figure 2A). We used a reporter composed of a fragment of Burdock expressed under the control of heterologous nanos promoter that drives expression in germline of both sexes (Handler et al., 2013). While reporter was efficiently silenced in ovaries of wild-type flies, it was strongly de-repressed in piRNA pathway mutants ( $\mathrm{rhi}^{-{ }^{--}}$) (Figure 3D), indicating that the piRNA program efficiently silences Burdock in female germline. In contrast, we observed strong reporter expression in testes of wild-type males, and the disruption of piRNA pathway in rhi mutants did not lead to an observable increase in its expression (Figure $3 \mathrm{C}$ ). This finding shows that Burdock is not silenced in testes, likely as a result of very few Burdock-targeting piRNAs in males (Figure 2A). Notably, expression of endogenous Burdock is high in ovary (when piRNA production is disrupted) but low in both wild-type and mutant testis (Figure 2E; Figure 3B). Thus, similar to telomeric TEs, the ability of piRNA pathway to repress Burdock in female but not male germline correlates with an intrinsic bias for its expression in females. We conclude that differential expression of TE-targeting piRNAs in male and female gonads can have functional consequences in their abilities to silence TEs, suggesting a sexually dimorphic TE-silencing piRNA program operating in the gonad.

\section{Definition of piRNA clusters in testis with a new algorithm}

To get deeper understandings of the piRNA program in male gonads, we sought to define the genomic origin of piRNAs and compare it between two sexes. Since genome-wide identification of piRNA clusters has only been done in ovary, we decided to systematically search for genomic 
loci that generate piRNA in testis. We noticed that two major clusters in testis identified to date, $\mathrm{Su}(\mathrm{Ste})$ and $A T$-chX, both contain internal tandem repeats, i.e., they are made of many copies of almost identical sequences (Aravin et al., 2001; Kotov et al., 2019). As a result, most piRNAs produced by these two loci mapped to the genome at multiple positions. However, the algorithm employed in previous studies to systematically define piRNA clusters in ovary only uses piRNAs that map to the genome at single unique positions (Brennecke et al., 2007; Mohn et al., 2014), raising the question of whether it is an appropriate approach to detect clusters like Su(Ste) composed primarily of internal tandem repeats. In fact, both $S u(S t e)$ and AT-chX clusters were initially identified by different approaches (Aravin et al., 2001; Nishida et al., 2007).

Even though piRNAs produced from $\mathrm{Su}(\mathrm{Ste})$ and $A T$-chX cannot be mapped to single unique genomic loci, most of them mapped to several local repeats inside the respective clusters but nowhere else in the genome (Figure 4A). Taking advantage of this property, we developed a new algorithm that takes into account local repeats to define piRNA clusters (Figure S4A and S4B). Briefly, in addition to uniquely-mapped piRNAs, the algorithm searches for piRNA sequences that map to multiple positions within a single genomic region but nowhere else in the genome. This approach ensures that the identified region as a whole generates piRNAs, though the exact origin within the region remains unknown. Unlike the previous approach that uses exclusively uniquely-mapped piRNAs, this algorithm successfully identified Su(Ste) and AT-chX, two major piRNA clusters in testis that contain local repeats (Figure 4E and 4F).

We applied this new algorithm to systematically identify piRNA clusters active in testes. We recovered piRNA clusters known to be active in testes as well as piRNA clusters previously defined in ovaries (e.g., 42AB, 38C, 20A and flam) (Figure 4C; Table S1). Furthermore, our search identified several novel piRNA loci. One of the novel piRNA clusters is located on $\mathrm{Y}$ chromosome flanked by FDY and Mst77Y genes (Figure 4C and 4F), which we called $h 17$ cluster using heterochromatin banding numbers (Gatti and Pimpinelli, 1983). Another novel locus is $h 52-1$, flanked by eIF4B and CG17514 genes on chr3L. h52-1 harbors tandem local repeats composed of nested TE fragments that cannot be found elsewhere in the genome. Similar to piRNA clusters identified in ovaries, only a few clusters active in testes produce piRNAs from one genomic strand (e.g., flam and 20A, so-called 'uni-strand clusters'), and the majority are dual-strand clusters that generate piRNAs from both genomic strands (Figure 4E). In sum, our algorithm successfully found previously known piRNAs clusters and identified novel ones in Drosophila testes.

To compare new algorithm with the approach that considers only uniquely-mapped piRNAs, we applied both techniques to analyze the same testis piRNA dataset. This comparison 
showed that major piRNA clusters in testis can be divided into two groups (Figure 4B). The first group (42AB, 38C, 20A and flam) contains piRNA clusters that harbor mostly unique sequences, so including local repeats or even all reads do not substantially change their identification and quantification. On the other hand, the second group of genomic loci (Su(Ste), AT-chX, h17, $H s p 70 B$ and $h 52-1$ ) is composed of piRNA clusters that contain many local repeats, and, accordingly, our new algorithm identified more than 10-fold more piRNAs produced from these loci (Figure 4B). Importantly, mapping of all piRNA reads (i.e., including multi-mappers not captured by our algorithm) only adds a negligible amount of piRNAs to these clusters, except for Hsp70B (Figure 4B). Thus, this algorithm is not only useful for finding new piRNA source loci but also provides a more accurate quantification of piRNA production from previously known clusters.

\section{Sex difference in piRNA cluster expression}

To compare the expression of piRNA clusters between sexes, we first applied our algorithm to published ovary piRNA datasets (Figure 4D; Table S1) (EIMaghraby et al., 2019). Thus, piRNA clusters were defined and their activities were quantified in both sexes using the same algorithm, allowing for fair comparison. Surprisingly, our analysis revealed that $A T$-chX, originally described as a piRNA cluster in testes, is also highly active in ovaries. AT-chX locus consists of local repeats (Kotov et al., 2019), so piRNAs produced from this locus were excluded in previous studies that analyzed only uniquely-mapped reads. In fact, $A T$-chX is the second most active piRNA cluster in ovary, producing $\sim 7 \%$ of total piRNAs.

Comparison between piRNA clusters in males and females revealed a clear sex difference: a small number of loci produce the majority of piRNAs in testis, which is not the case for ovary (Figure 5A). The two most active piRNA clusters in testes, Su(Ste) on $\mathrm{Y}$ chromosome and ATchX on $X$ chromosome, produce $\sim 43 \%$ and $\sim 31 \%$ of total piRNAs in testes, respectively (Figure $4 C)$. They are followed by the novel piRNA cluster on $Y$ chromosome, $h 17$, that produces $\sim 4 \%$ piRNAs. Along with another 6 loci, the top 9 piRNA clusters in testis account for $81.8 \%$ of total piRNAs. In comparison, only $30.4 \%$ of total piRNAs are made from the top 9 clusters in ovary, with the most active locus $42 A B$ producing $\sim 11 \%$ of total piRNAs (Figure $4 \mathrm{D}$ ). Whereas a few loci dominate the global piRNA population in testis, the ovary piRNA profile is shaped by many loci producing piRNAs in comparable amounts.

Next, we compared expression levels of different piRNA clusters in male and female gonads. Females lack Y chromosome, so they do not have piRNAs produced by Y-linked Su(Ste) 
and $h 17$ clusters. For major clusters present in both male and female genomes, we observed pronounced sex differences (Spearman's $\rho=0.07$; Figure 5B). For instance, 38C produces more piRNAs than $42 A B, 80 E F$ and $40 F 7$ in testes, but the opposite trend is found in ovaries. Some loci such as Sox102F on chr4 (Mohn et al., 2014; Zhang et al., 2014) appear to be active only in ovaries but not in testes (Figure 5F). These differentially expressed piRNA clusters located on autosomes, which both males and females have two copies, exemplify the sex-specific usage of piRNA loci. Moreover, we examined expression levels of major piRNA clusters on $\operatorname{ch} r(A T$-chX, flam and 20A), which females have two copies (XX) and males have only one (XY). We found that a larger fraction of piRNAs originate from $A T$-chX in testes than ovaries, but the reverse was found for flam and 20A, suggesting that copy numbers of piRNA clusters do not correlate well with their expression. Altogether, these findings illustrate a sexually dimorphic employment of piRNA clusters, where different loci are engaged differentially in a sex-specific manner.

Different piRNA clusters have distinct TE contents, so their differential expression might sculpt sex-specific piRNA programs with distinct TE-silencing capacities in males and females. To explore a link between the expression of piRNA cluster and its TE content, we computed cumulative sex bias of the TE content of each major piRNA cluster (Figure 5C). This was done by summing sex biases of individual TEs in the piRNA cluster weighted by their length contributions to the cluster (see example in Figure $5 \mathrm{C}$ ). The sex bias of cluster TE content matches the sex bias in piRNA cluster expression, suggesting a link between the expression of piRNA clusters and TEs they control. To substantiate this finding, we analyzed sequence compositions of three differentially expressed piRNA clusters: $42 A B$ (ovary-biased), 38C (testisbiased) and Sox102F (ovary-specific). The top 6 TEs most enriched by length in ovary-biased $42 A B$ (batumi, gypsy12, FW, DMRT1b, copia2 and max) are all ovary-biased in their expression (Figure 5D; Figure S3A). Importantly, these 6 TEs are completely absent in testis-biased 38C cluster. In contrast, three testis-biased TE families, hobo, BS2 and Transib2, are more enriched in $38 C$ than in $42 A B$ (Figure 5E; Figure S3A). Moreover, ovary-specific Sox102F cluster harbors a single autonomous transposon, Tc1-2, which has higher activity in ovary (Figure 5F; Figure S3A). These examples show that differential expression of piRNA clusters in two sexes often matches the differential activities of TEs they control, supporting the notion that piRNA clusters are employed in a sex-specific fashion to cope with distinct TE landscape in male and female gonads.

\section{piRNA clusters composed of local repeats produce piRNAs that target host genes}


Our analysis indicated that $13.8 \%$ of testis piRNAs might potentially be involved in targeting host genes as they can be mapped to protein-encoding genes in antisense orientation with a small number ( 0 to 3 ) of mismatches between piRNA and gene sequences (Figure 1B). To understand the genomic origin of these piRNAs, we further analyzed sequence compositions of piRNA clusters. We found that two clusters, $H s p 70 B$ and $h 17$, both of which contain local repeats, generate piRNAs that have the potential to target host genes.

The $H s p 70 B$ cluster spans $\sim 35 \mathrm{~Kb}$ between two paralogous $H s p 70 B$ genes on chr3R, and it is active in both ovary and testis (Figure 6A). The body of Hsp70B cluster contains several TEs. Even though there are piRNAs mapping to these TEs, they can be mapped elsewhere in the genome as well, rendering it impossible to be certain that they originate from Hsp70B locus. In fact, this cluster was previously identified through the presence of uniquely-mapped piRNAs from flanking non-repetitive genes (Mohn et al., 2014). However, our algorithm that takes into account local repeats revealed piRNAs generated from a $\sim 354 \mathrm{bp}$ local repeat at $H s p 70 B$ locus, which occupies nearly all inter-transposon space within this cluster. Importantly, these piRNAs mapped exclusively to this local repeat at $H s p 70 B$ cluster but nowhere else in the genome. Intriguingly, these repeats have a $\sim 92 \%$ sequence identity to an exon of the nod gene, which encodes a kinesin-like protein necessary for chromosome segregation during meiosis (Carpenter, 1973; Hawley and Theurkauf, 1993; Zhang et al., 1990). Hsp70B cluster generates piRNAs that are antisense to nod with a $91.3 \%$ averaged nucleotide identity to it. This level of sequence similarity is close to that between Suppressor of Stellate piRNAs and their Stellate targets, the first known case of piRNA repression (Aravin et al., 2001; Vagin et al., 2006), suggesting that piRNAs produced from $H s p 70 B$ locus might be able to repress the nod gene.

The second locus producing piRNAs that might target host genes is the novel piRNA cluster $h 17$ on $\mathrm{Y}$ chromosome, which is only present in $\mathrm{XY}$ males (Figure 6B). This cluster spans more than $200 \mathrm{~Kb}$ and includes two loci duplicated from chr2L and chrX, respectively, that contain almost the entire CG12717 gene (which encodes a SUMO protease) and small parts of Paics (which encodes an enzyme involved in purine biogenesis) and ProtA (which encodes protamine, a sperm chromatin protein) (Mendez-Lago et al., 2011). These gene-homologous sequences are further duplicated locally on $Y$ to over 20 copies and take up nearly all space in between TEs at h17 cluster (Figure 6B; Figure S5B). However, these gene-related sequences likely do not retain coding potentials as they are frequently interrupted by TE sequences. $h 17$ cluster produces piRNAs antisense to CG12717, Paics and ProtA genes, with averaged levels of nucleotide identity 
92.5\%, 93.9\%, and 91.0\%, respectively. Together, two piRNA clusters, $H s p 70 B$ and $h 17$, encode piRNAs with the potential to target both TEs and host genes.

We quantified expression of piRNAs antisense to nod, CG12717, Paics and ProtA genes from these two clusters. Even though these piRNAs all possess over $90 \%$ identity to their putative targets, their abundances differ dramatically (Figure 6C). CG12717 gene is targeted by abundant piRNAs (4,310 RPM), comparable to the $15^{\text {th }}$ most targeted TE family in testis. piRNAs against nod are expressed at 813 RPM ( 5-fold less compared to CG12717), while the levels of piRNA against Paics or ProtA are low (both $\sim 50$ RPM). In addition, nearly the entire length of CG12717 gene is targeted by piRNAs, whereas only small parts of nod, Paics and ProtA are targeted. These findings suggest that CG12717 and nod might be regulated by piRNAs in testis.

\section{piRNA-guided repression of SUMO protease CG12717/pita during spermatogenesis}

To examine the role of piRNAs in gene regulation, we employed RNA-seq to analyze expression of host genes in testes of three different piRNA pathway mutants: aub, zuc and spn-E (Nishida et al., 2007; Pane et al., 2007; Schmidt et al., 1999; Stapleton et al., 2001). Transcriptome profiling revealed that only two genes, CG12717 and frtz, exhibited $\geq 2$-fold up-regulation in all three piRNA pathway mutants (Figure 7A). Unlike CG12717, there are very few, if any, antisense piRNAs targeting frtz, so its up-regulation likely reflects a secondary phenotype following TE de-repression. Strikingly, expression of CG12717 increased more than 10-fold in all three mutants (Figure 7C), indicating that it is indeed strongly repressed by the piRNA pathway. Meanwhile, we observed no statistically significant up-regulation of nod, Paics or ProtA in these three mutants (Figure 7C), correlating with fewer piRNAs against these genes than CG12717 (Figure 6C). While CG12717 is expressed at a very low level in testes of wild-type males, it is highly expressed in ovaries (Figure 7D), consistent with the fact that CG12717-targeting piRNAs are encoded on $Y$ chromosome. Thus, transcriptome profiling identifies CG12717 as a target of piRNA repression and suggests that abundant antisense piRNAs with high target coverage might be required for efficient silencing.

piRNA-guided cleavage of target RNAs often triggers the production of secondary piRNAs from target RNAs in a process dubbed ping-pong cycle (Brennecke et al., 2007). Examination of piRNA sequences revealed abundant piRNAs derived from the entire length of CG12717 mRNAs (Figure 6C). In contrast, we found few piRNAs processed from transcripts of nod, Paics or ProtA. Furthermore, sense piRNAs derived from CG12717 mRNAs and antisense piRNAs produced 
from $h 17$ cluster demonstrated a strong ping-pong signature $\left(Z_{10}=16.8\right.$; Figure $\left.7 \mathrm{E}\right)$, characteristic of active ping-pong cycle. This finding further suggests the direct cleavage of CG12717 transcripts guided by $h 17$ piRNAs. Finally, we performed RNA in situ hybridization chain reaction (in situ HCR) to examine CG12717 expression. Expression of CG12717 is very low in wild-type testis, but it was significantly increased in testes of $a u b, z u c$ and spn-E mutants (Figure 7B). Upon release of piRNA silencing in these three mutants, CG12717 is specifically expressed in differentiating spermatocytes, but not in germline stem cells or mitotic spermatogonia. Interestingly, Stellate is expressed at the same stage when the silencing by $S u(S t e)$ piRNAs is removed (Aravin et al., 2004). As our results indicate that expression of CG12717, a SUMO protease gene related to Ulp2 in yeast (Berdnik et al., 2012), is strongly repressed by piRNAs, we propose to name it pita (piRNA target).

To understand how piRNA-dependent regulation of pita has evolved, we performed a blastN search using Drosophila melanogaster pita gene against genomes of other Drosophila species. We found multiple copies of pita-related sequences in genomes of Drosophila simulans species complex (D. simulans, D. sechellia and D. mauritiana) (Figure 7F), but not in more distantly related species like $D$. erecta or $D$. yakuba. Similar to $h 17$ cluster in $D$. melanogaster, these pita-related sequences reside in TE-rich regions (either peri-centromeric heterochromatin or unassigned scaffolds) in $D$. simulans species complex. While all pita-related sequences are exclusively located at $h 17$ on $Y$ chromosome of $D$. melanogaster, pita-homologous sequences can be found on different chromosomes in genomes of $D$. simulans species complex. For instance, in D. mauritiana, pita-homologous sequences can be found on at least chrY, chrX, chr3L and chr3R (Figure 7G). Therefore, duplications of pita-related sequences into heterochromatin have occurred in all four species.

To investigate whether heterochromatic pita-homologous sequences produce small RNAs in testes of other species, we analyzed published small RNA datasets from testes of $D$. simulans and D. mauritiana (Kotov et al., 2019; Lin et al., 2018). We found no small RNAs mapping to the orthologous pita gene in $D$. simulans testes, but abundant ones in D. mauritiana testes (Figure $7 \mathrm{H})$. Unexpectedly, unlike 23-29nt pita-mapping piRNAs in D. melanogaster, pita-mapping small RNAs in $D$. mauritiana are mostly $21 \mathrm{nt}$ long, indicating that they are endo-siRNAs. These endosiRNAs have on average $93.5 \%$ identity with the $D$. mauritiana pita gene. Notably, similar to other dual-strand piRNA clusters described in D. melanogaster ovaries (Czech et al., 2008; Le Thomas et al., 2014), h17 cluster in D. melanogaster testes also generates pita-mapping endo-siRNAs, though much less abundant than 23-29nt piRNAs (Figure $7 \mathrm{H}$ ). Examination of heterochromatic, 
pita-homologous sequences in $D$. mauritiana genome revealed that most of them are arranged head-to-tail (Figure 7G). However, there are four instances where pita-homologous sequences are arranged head-to-head (Figure $7 G$ and $7 \mathrm{I}$ ), which could potentially generate hairpin RNAs (hpRNAs), the preferred substrate for processing into endo-siRNAs by Dicer. Thus, targeting of pita by small RNAs in testis seems to be conserved in two Drosophila species. While pita is repressed mostly by piRNAs in $D$. melanogaster, it is targeted nearly exclusively by endo-siRNAs in $D$. mauritiana, suggesting two related but distinct regulation strategies employed in sibling species that diverged less than 5 million years ago.

In addition to pita, there is another SUMO protease, veloren (velo) in D. melanogaster genome. pita and velo are paralogs whose homologous domains share $75 \%$ nucleotide identity (Figure S5A). In agreement with the sequence similarity, functions of Pita and Velo in SUMO deconjugation pathway were shown to be partially redundant (Berdnik et al., 2012). Phylogenetic analysis showed that, while velo is found at syntenic locations on chromosome 3 throughout the Drosophila genus, pita is much younger and was only born after the split of $D$. melanogaster and ananassae species subgroups (Figure 7F). These results indicate that pita and velo have evolved from a common ancestor gene, via inter-chromosomal duplication from chr3 to X chromosome.

Considering the $75 \%$ nucleotide identity between the parts of pita and velo genes in $D$. melanogaster, pita-targeting piRNAs produced from $h 17$ cluster have a potential to target velo transcripts. However, we found that none of the pita-antisense piRNAs can be mapped to velo transcript perfectly. Moreover, $\sim 200$-fold fewer piRNAs have a potential to target velo with one to three mismatches. Transcriptome profiling in testes of aub, zuc and spn-E mutants showed that, unlike pita, velo is not repressed by piRNAs (Figure 7C). In addition, while pita is only expressed in ovaries, velo is expressed in both testes and ovaries and, in fact, has a higher expression level in testes (Figure 7D). These results show that $Y$-linked $h 17$ piRNAs repress specifically pita, but not its paralog, velo, suggesting that a high degree of complementarity is required for efficient piRNA silencing. Therefore, piRNAs distinguish closely related paralogs to achieve sex- and paralog- specific gene regulation.

Taken together, our results allowed us to reconstruct the evolutionary history of two paralogous, Ulp2-like SUMO protease genes. First, the pita gene was born via inter-chromosomal duplication after the split of $D$. melanogaster and ananassae species subgroups. This then permitted the differentiation of velo and pita functions, though these two genes remain in part functionally redundant in D. melanogaster (Berdnik et al., 2012). Next, divergence between pita and velo sequences created an opportunity for paralog-selective gene regulation by small RNA- 
guided mechanisms. This was achieved by duplications of pita sequences into heterochromatin in genomes of $D$. melanogaster and $D$. simulans species complex. It is plausible that, initially, heterochromatic, pita-homologous sequences did not play a role in gene regulation, as illustrated by the absence of pita-mapping small RNAs in D. simulans. However, subsequent expansion and interaction with TE sequences might have enabled the evolution of two distinct repression mechanisms, via production of pita-targeting piRNAs and endo-siRNAs, that dominated in $D$. melanogaster and D. mauritiana, respectively. Repression of pita by $Y$-linked piRNAs led to its specific repression in $D$. melanogaster testis, implicating the piRNA pathway in establishing distinct expression patterns of closely related paralogs after gene duplication.

\section{DISCUSSION}

Previous studies systematically analyzed piRNA profiles in female gonads of $D$. melanogaster, revealing an essential role of piRNAs in regulation of many TEs (Brennecke et al., 2007; Li et al., 2009; Malone et al., 2009). However, these studies only provided a single snapshot of the relationship between TE and piRNA defense system, as they are insufficient to understand how the piRNA program might adapt to changing TE repertoire and different levels of their expression. To this end, several studies explored the piRNA pathway in other species of Drosophila (Malone et al., 2009; Rozhkov et al., 2010; Saint-Leandre et al., 2020). These studies revealed that piRNA profiles are different across species, suggesting an adaptation of the defense mechanism to distinct challenges. However, drastic differences in both TE contents and piRNA cluster sequences even among closely related Drosophila species (Kofler et al., 2015; Lerat et al., 2011; Malone et al., 2009) make it difficult to disentangle different factors that sculpt species-specific piRNA programs. Here, we examined TE expression in males and females of the same species, revealing strong differences in TE activities between sexes. This allowed us to compare piRNA programs in two sexes with similar genomic contents (except $Y$ chromosome).

Another obstacle to understanding responses of the piRNA program to TEs is properly assessing TE expression. D. melanogaster genome includes over 100 different TE families whose expression levels can be measured by standard methods such as RNA-seq. However, TE expression in wild-type animals is greatly suppressed by the piRNA pathway (>100-fold for some families) (EIMaghraby et al., 2019). Therefore, in order to understand true expression potentials of TEs, it is necessary to study their expression upon removal of piRNA silencing, which is difficult to do in species other than model organisms like $D$. melanogaster. In this work, we examined the TE expression in piRNA pathway mutants, revealing genuine potentials of TE expression in both 
sexes. Combined analysis of TE and piRNA expression showed responses of the piRNA program to distinct TE expression profiles in two sexes.

Analysis of the genomic origin of piRNAs represents an important but challenging task. As piRNA sequences are short (23-29nt) and often derive from repetitive genomic regions, a large fraction of sequenced piRNA reads can be mapped to multiple genomic loci, preventing an unambiguous assignment of their origin. Accordingly, algorithms employed in previous studies only used the small fraction of piRNA reads that can be mapped to the genome at single unique positions to identify genomic regions that generate piRNAs. We took advantage of the fact that some genomic repeats are local, i.e., they reside within one genomic region and are absent in the rest of the genome, to develop a new algorithm for piRNA cluster definition and analysis (Figure 4A and Figure S4). This approach was successful in identifying new piRNA clusters. Furthermore, it also provided a more accurate quantification of the piRNA cluster expression. We found that Hsp70B cluster generates piRNAs against the nod gene. In addition, we discovered a novel cluster, $h 17$, on $\mathrm{Y}$ chromosome that generates piRNAs against three host genes and ensures the strong silencing of SUMO protease, CG12717/pita, during spermatocyte differentiation.

Our identification of the novel $h 17$ cluster on $\mathrm{Y}$ expanded known functions of entirely heterochromatic $\mathrm{Y}$ chromosome (Figure 4F and Figure 6B). Three functionalities have been assigned to $Y$ by the early 1980s (Gatti and Pimpinelli, 1983). First, together with $X$ chromosome, $Y$ encodes rDNA loci that express rRNAs and mediates meiotic pairing with $X$. Second, $Y$ encodes six protein-encoding genes, so-called "fertility factors", whose protein products are required for completion of spermatogenesis. Finally, Y chromosome harbors the Su(Ste) locus that generates piRNAs to suppress Stellate genes to safeguard normal spermatogenesis (Aravin et al., 2001; Vagin et al., 2006). A handful of new protein-encoding genes were discovered on $Y$ in the past two decades (Bernardo Carvalho et al., 2009; Krsticevic et al., 2010), however, many of them appeared dispensable. Our finding that $Y$ chromosome encodes a novel piRNA cluster and produces piRNAs to regulate expression of the pita gene assigns a new function to $\mathrm{Y}$ chromosome.

\section{Sexual dimorphism of TE expression and TE-silencing piRNA programs}

D. melanogaster is an excellent model to study TE regulations and host-TE interactions, as its genome harbors many TE families that are transcriptionally and transpositionally active, generating new insertions in the population (Kofler et al., 2015). Our results indicate that expression of both TEs and piRNAs is sexually dimorphic. The majority of TE families are strongly 
expressed in ovaries, though some TEs are more active in testes. In line with this, our results indicate a stronger TE-silencing piRNA program in female gonads (Figure 2).

For TEs to be evolutionarily successful, they need to evolve strategies to maximize their chance to be inherited and expanded through generations. For example, TEs often hijack germline gene expression programs to be preferentially active in germ cells. Germline-biased expression leaves the choice of expression to either female or male germline, or both. Importantly, the two sexes employ distinct evolutionary strategies and have different contributions towards the zygote. While the major contribution of sperm is its genome, oocyte contributes large amounts of yolk, various protein factors, RNAs and organelles such as mitochondria, in addition to its genome. This sexual asymmetry in their contributions to the next generation has important implications for reproduction strategies of TEs. TEs active in the male germline need to complete the entire life cycle from transcription to genomic insertion before sperm maturation, in order to propagate. In contrast, once transcribed, TEs active during oogenesis could finish their life cycle in the zygote after fertilization, as long as transcribed TE transcripts are deposited into the oocyte. The latter strategy is also used by mammalian L1 retrotransposon that is expressed during gametogenesis, but genomic insertions might occur later during early embryogenesis (Kano et al., 2009). Thus, the expression bias towards ovaries observed for most TEs can be explained by an advantage for their proliferation, specifically, the extended window to finish their life cycle, in female germline.

There are a few TEs that bias testis for expression, suggesting that there are likely malespecific vulnerabilities exploitable by these elements. For example, male germ cells use a testisspecific gene expression machinery (e.g. tTAF and tMAC) to transcribe meiotic and post-meiotic genes (Beall et al., 2007; Hiller et al., 2004). TEs might exploit this tissue-specific transcriptional machinery to enable their sex-biased expression. It will be important in the future to uncover molecular mechanisms underlying differentially expressed TEs between sexes.

Analysis of piRNA profiles in testis and ovary indicates that piRNA programs have adapted to sex-biased TE expression (Figure 3). The most striking example is the nearly exclusive expression of telomeric TEs and corresponding antisense piRNAs in the female germline. Our results suggest that differential expression of piRNA clusters in two sexes together with differential TE-targeting capacity of each cluster contributes to the sex-specific, TE-targeting piRNA program. We found that piRNA cluster expression is sexually dimorphic. Besides the Su(Ste) locus, we identified another major cluster on $Y$ chromosome that is only active in $X Y$ males. However, sexbiased expression is not restricted to $Y$-linked clusters, as many $X$-linked and autosomal clusters have differential activities between sexes as well. Besides differential expression, genomic 
analysis showed differences in piRNA cluster TE contents, suggesting that different piRNA clusters are, to some extent, specialized to target different sets of TEs. Importantly, sex bias in cluster expression and their TE-targeting potentials are linked: clusters preferentially targeting ovary-biased TEs are more active in ovary, while testis-biased clusters tend to target testis-biased TEs (Figure 5). Hence, piRNA clusters appear to be employed differentially by two sexes to counteract specific TE threats they face. What determines the differential expression of piRNA clusters between sexes awaits future studies. Previous work suggests that TE promoters embedded in piRNA clusters retain their activities (Mohn et al., 2014). Contribution of TE promoters to piRNA precursor transcription from piRNA clusters might explain the correlation between expression of clusters and their TE targets.

\section{Satellite DNA as target of piRNA silencing}

Satellite DNAs can be classified as either simple or complex satellites based on the length of repeating units, and they occupy large portions of Drosophila genome, particularly at pericentromeric and sub-telomeric regions (Hsieh and Brutlag, 1979; Karpen and Spradling, 1992; Larracuente and Presgraves, 2012; Lohe et al., 1993). We found piRNAs expressed from three major families of complex satellites: sub-telomeric HETRPITAS, Responder (Rsp), and $S A R / 1.688$ (including 359bp). In fact, piRNAs can be mapped to both strands of complex satellites in gonads of both sexes, and they often possess ping-pong signature (Figure 1C). Thus, our results expand the previous observation of piRNAs mapping to one strand of Rsp (Saito et al., 2006) and establish complex satellites as dual-strand piRNA clusters and potential targets of piRNA silencing in Drosophila germline of both sexes. Our analysis was focused on complex satellites, as simple satellite repeats are still largely intractable to sequencing technologies today (Khost et al., 2017). However, a recent study reported that transcripts from AAGAG simple satellite repeats regulate heterochromatin in male germline and are required for male fertility (Mills et al., 2019). It will be interesting to determine whether simple satellites produce piRNAs and, if so, whether their piRNA production is required for male fertility.

piRNAs loaded onto the nuclear Piwi protein guide heterochromatin assembly (Le Thomas et al., 2013; Rozhkov et al., 2013; Sienski et al., 2012; Wang and Elgin, 2011). For this reason, satellite piRNAs might play a role in establishing germline heterochromatin, similar to heterochromatin formation guided by siRNAs in fission yeast (Hall et al., 2002; Volpe et al., 2002). While the function of complex satellites remains mostly elusive, $R s p$ has been implicated in a meiotic drive system called segregation distortion (Hartl, 1973; Larracuente and Presgraves, 2012; 
Wu et al., 1988). During male meiosis, the Segregation Distorter (SD) allele enhances its own transmission to haploid cells at the cost of wild-type $\left(S D^{+}\right)$allele in $S D / S D^{+}$heterozygous males, violating Mendelian law of inheritance. Importantly, segregation distortion requires the presence of a sufficient number of Rsp satellite repeats in trans. Though described more than 60 years ago (Sandler et al., 1959), the molecular mechanism of segregation distortion remains unknown. Intriguingly, mutations of aubergine (aub), a PIWI protein, were found to be enhancers of SD (Gell and Reenan, 2013). Together with our data, these data suggest that piRNA pathway may play a role in segregation distortion during spermatogenesis.

\section{Regulation of host genes by piRNAs}

Though the central and conserved function of piRNA pathway seems to be TE repression, other functions were also described in several organisms (reviewed in Ozata et al., 2019). The role of piRNAs in regulating host gene expression is particularly intriguing and remains somewhat controversial. The first described piRNAs, Su(Ste) piRNAs, silence the expression of Stellate genes (Aravin et al., 2001; Vagin et al., 2006). However, Stellate genes and their piRNA suppressors appear to resemble selfish toxin-antitoxin systems rather than representing an example of host gene regulation (Aravin, 2020). Since the discovery of piRNA pathway, there have been several studies reporting host protein-encoding genes regulated by Drosophila piRNAs (reviewed in Rojas-Ríos and Simonelig, 2018). In this work, we analyzed the ability of Drosophila piRNAs to regulate host genes in testes, by examining gene-targeting piRNAs and changes in host gene expression across three piRNA pathway mutants. We found piRNAs targeting four host genes: nod (a kinesin-like protein), CG12717/pita (a SUMO protease), Paics (a metabolic enzyme) and $\operatorname{ProtA}$ (a sperm chromatin protein). These four genes are targeted by antisense piRNAs generated from two piRNA clusters that contain sequence homology to them. However, only one of the four, CG12717/pita, is substantially repressed (over 10-fold) by piRNAs (Figure 7). As pitasilencing piRNAs are encoded on $Y$ chromosome and thus only expressed in males, they are responsible for differential expression of pita in two sexes. Indeed, in wild-type files, pita is specifically silenced in male gonads while highly expressed in female counterparts. Thus, our results establish the ability of piRNAs to repress host protein-encoding genes, and, at the same time, suggest that this role is likely restricted to a small number of genes.

Our results indicate that piRNA-guided repression of host genes requires a sufficient number of targeting piRNAs. While all four genes are targeted by piRNAs with similar levels of sequence identity (91-94\%, i.e., about 2 mismatches per piRNA), the abundance of piRNAs 
against each gene differs drastically. There are much more pita-targeting piRNAs than the other three gene targets, at a level comparable to the $15^{\text {th }}$ most targeted TE. Furthermore, while pita is targeted along almost the entire length, only small regions of other three genes are targeted by piRNAs. These differences in piRNA abundance and distribution of target sites could explain strong repression of pita, in contrast to the other three genes. It is possible that these genes are still regulated by piRNAs at specific stages, the question that remains to be further investigated. Importantly, abundant pita-silencing piRNAs do not repress the pita paralog, velo, that has a $75 \%$ sequence identity with pita, indicating that a high complementarity between piRNA and target may be important for efficient silencing. In agreement with these, a previous report indicated that a similar level of sequence identity $(\sim 76 \%)$ is insufficient for the silencing of vasa by $A T$-chX piRNAs (Kotov et al., 2019). Therefore, both high expression and high complementarity with targets might be required for efficient piRNA silencing in $D$. melanogaster.

This conclusion is important for analyzing the potential of piRNAs to repress host proteincoding genes. Unlike miRNAs, sequences of piRNAs are extremely diverse. Accordingly, if mismatches between piRNA and its target are well tolerated, a large number of cellular mRNAs should be targeted and repressed by piRNAs. Indeed, some host genes were proposed to be repressed by a few piRNA species that have multiple mismatches to mRNA sequences (Gonzalez et al., 2015; Klein et al., 2016; Rojas-Ríos et al., 2017; Saito et al., 2009). Our results suggest that such a spurious targeting by individual piRNAs is unlikely to cause repression. In fact, a high threshold for efficient target repression might permit production of diverse piRNA sequences against genuine targets such as TEs, without unintended interference with host gene expression.

\section{The role of piRNA in evolution}

Analysis of pita repression revealed a remarkable picture of evolutionary innovation (Figure 7). piRNA-dependent repression of pita occurs in D. melanogaster but not in its sibling species, suggesting its rather recent origin. Efficient silencing of pita is linked to the presence of multiple copies of pita-homologous sequences in a piRNA cluster inside heterochromatin. Interestingly, duplications of pita sequences into, and their expansion within, heterochromatin can be found in three closely related species of $D$. simulans complex, in addition to $D$. melanogaster. However, distribution and copy number of pita-related sequences differ among these four species. In fact, both $h 17$ locus that generates pita-silencing piRNAs and its two flanking protein-encoding genes, FDY and Mst77Y, evolved after the split of $D$. melanogaster and $D$. simulans species complex (Carvalho et al., 2015; Krsticevic et al., 2010; Mendez-Lago et al., 2011), suggesting that the 
entire locus is unique to $D$. melanogaster. Furthermore, no small RNAs are generated from heterochromatic pita sequences in $D$. simulans, while endo-siRNAs are made against pita in $D$. mauritiana. The neutral theory of molecular evolution provides the most parsimonious interpretation of these results. This theory suggests that the initial duplication of pita sequences into heterochromatin might have been a random event that did not play a role in regulating the ancestral pita gene. However, subsequent evolution of pita-related sequences inside heterochromatin gave rise to two different modes of regulations, piRNA and endo-siRNA, in two different but closely related species. The emergence of small RNA-mediated repression was probably facilitated by the fact that pita itself was recently evolved and retains partially redundant functions with its paralog, velo (Berdnik et al., 2012), allowing independent regulation of two paralogs.

The evolutionarily innovative role of piRNAs in regulating host genes in Drosophila has interesting parallels in other organisms. Pachytene piRNAs expressed during spermatogenesis in mammals evolved very fast and are generally poorly conserved (Özata et al., 2020). The function of pachytene piRNAs is under active debate as no obvious targets can be easily discerned by analysis of their sequences (Aravin et al., 2006; Girard et al., 2006). Recently, knockout of one pachytene piRNA cluster led to unexpected conclusion that a small fraction of piRNAs promote biogenesis from other piRNA clusters and regulate the expression of a few host genes, while the vast majority do not target any transcripts (Wu et al., 2020). Thus, mammalian pachytene piRNAs can be considered a selfish system that occasionally involves in regulation of the host gene expression. Species-specific regulation of host genes by piRNAs in both Drosophila and mouse suggests that piRNA pathway is used in evolution to create innovation in gene regulatory networks that might contribute to speciation. More generally, piRNAs might promote the evolvability of animal species. Though it is difficult to establish the function of any molecular mechanism in evolution, this proposal makes a testable prediction that host genes repressed by piRNAs differ even among closely related species. Future studies in non-model organisms will shed light on the role of piRNAs in evolution and speciation. 


\section{ACKNOWLEDGEMENT}

We are grateful to William Theurkauf, Trudi Schüpbach, Julius Brennecke and Bloomington Drosophila Stock Center for fly stocks. We thank Katalin Fejes Toth and members of Aravin lab for discussion and comments. We appreciate the help of Maria Ninova and Fan Gao (Bioinformatics Resource Center, Caltech) with bioinformatics analysis, the help of Grace Shin and Maayan Schwarzkopf with HCR experiments, the help of Giada Spigolon and Andres Collazo (Biological Imaging Facility, Caltech) with microscopy, and the help of Igor Antoshechkin (Millard and Muriel Jacobs Genetics and Genomics Laboratory, Caltech) with sequencing. This work was supported by grants from the National Institutes of Health (R01 GM097363) and by the HHMI Faculty Scholar Award to A.A.A.

\section{DECLARATION OF INTERESTS}

The authors declare no competing interests. 


\section{MAIN FIGURE TITLES AND LEGENDS}

Figure 1. Analysis of small RNA profiles in testis and ovary.

(A) Size distribution plots of microRNAs (gray), remaining small RNAs that map to TE consensus (red) and protein-encoding gene exons (black), in testis (left) and ovary (right).

(B) Annotation of piRNA reads in testis (left) and ovary (right). $1 \mathrm{U}$ nucleotide bias (\%) for overall piRNA population and each category is shown next to labels. See also Figure S1.

(B) Characterization of piRNAs mapping to three known complex satellites in two sexes. Left panels of each sex are size distribution of piRNAs mapping to consensus sequences of each complex satellite. Right panels are distributions of 5'-to-5' distances of piRNA pairs, showing an enrichment for 10nt (i.e., ping-pong signature), except for Rsp in testis. 1 U nucleotide bias (\%) and ping-pong z-score are shown above plots. See also Figure S2.

\section{Figure 2. Expression of piRNAs and TEs are both sexually dimorphic.}

(A) Heatmaps showing the abundance of antisense piRNA (left) and ping-pong z-score (right) for each TE family in two sexes. TE families are sorted by sex bias of piRNA expression, defined as the log2 ratio of antisense piRNA abundance in testis over ovary. TEs with more than 2-fold differences in antisense piRNAs are colored as testis-biased (blue) and ovary-biased (pink), respectively, with the remaining having no obvious bias (gray).

(B) Top 10 TEs targeted by the most abundant antisense piRNAs in testis (left) and ovary (right). Heights of slices correspond to relative abundance in each sex, and the sum of top 10 TEs is then scaled to the same height between sexes. Each TE family is given a unique color, and the same TE family is connected by a line to help visualize distinct rank-orders between sexes. Names of TE families are shown following the same order, though not directly next to respective slices.

(C) Top 10 most expressed TE families in piRNA pathway mutant testis (left) and ovary (right). $\mathrm{rhi}^{-/-}$was used, where piRNA production from genome-wide dual-strand piRNA clusters collapses. Slice heights and colors were depicted as described in (B), though the same TE can be marked by a different color from (B).

(D) Scatter plot displaying the fold-change of TE expression in piRNA pathway mutant ( $\left.r \mathrm{i}^{-{ }^{-}}\right)$testis (left, blue) and ovary (right, pink) over controls. Venn diagrams of the number of TEs showing 100-, 10- and 4-fold de-repression in two sexes are shown on the right. 
(E) Expression of 36 TE families that are regulated by rhi (see methods) in testis (left) and ovary (right). TE families are sorted by sex bias of their expression in piRNA pathway mutant $\left(r h i^{-{ }^{-}}\right)$, defined as the log2 ratio between sexes. Heatmaps display TE levels in control and mutant, while bar graphs show the fold-change of expression in mutant over control.

Figure 3. Sexually dimorphic piRNA programs parallel sex-specific TE expression.

(A) Scatter plot displaying the correlation between sex biases of TE and TE-antisense piRNA. For each TE family, the loss of antisense piRNAs in rhi mutants was calculated in each sex (ctrl RPM over mut RPM). The sex bias of piRNAs was defined as the log2 ratio of piRNA loss in female over male. Similarly, TE de-repression in rhi mutants was calculated in each sex (mut RPM over ctrl RPM), and the sex bias was defined as the log2 ratio of TE de-repression in female over male. TE families that show a correlation between the sex bias of antisense piRNA and that of TE derepression are colored as orange, with the rest as blue.

(B) Histograms showing profiles of two sex-biased TEs for each sex. Antisense piRNA levels refer to those in control gonads, TE levels refer to those in piRNA pathway mutants $\left(r h i^{-/}\right)$, and the foldchange is calculated as mutant over control for TEs and the reverse for antisense piRNAs.

(C) Confocal images of the apical tip of testis (left) and stage 7-8 nurse cells in ovary (right) that express a Burdock-fused GFP reporter. The reporter is expressed by nanos promoter that drives germline expression in both sexes, thus enabling the examination of piRNA silencing of Burdock sequences independent of natural expression patterns of Burdock transposon. Scale bars: $20 \mu \mathrm{m}$.

\section{Figure 4. Definition of piRNA clusters in testis and ovary using a new algorithm.}

(A) Three types of piRNA reads, defined based on their mapping positions. Uniquely-mapped reads can be mapped to only one position in the genome and their origin is unambiguous. Reads derived from local repeats can be mapped to several positions in the genome; however, all of these mapping positions are locally clustered in a single genomic region. On the other hand, nonlocal multi-mappers can be mapped to multiple positions that are not restricted to one genomic region (typically mapped to more than one chromosome). Previously, only uniquely-mapped reads were used to define piRNA clusters and quantify their expression, as the genomic origin of multi-mappers is ambiguous. Inclusion of multi-mappers derived from local repeats, as shown in this study, allows identification of new piRNA clusters as well as a more accurate quantification 
of piRNA production from known clusters. At the same time, it preserves the certainty that reads are generated from genomic loci in question. See Figure $S 4$ for detailed pipeline.

(B) Histogram comparing numbers of mapped reads for major piRNA clusters using different readinclusion criteria as defined in (A). For each cluster, the number of mapped reads generated by different methods is normalized to the method that includes both unique and local repeat reads (the middle column). See also Figure S4 and methods.

(C) Expression of the top 9 most active piRNA clusters in testis. Blue bars depict the contribution of each cluster to total piRNAs (\%) and orange dots show cluster lengths according to dm6 genome assembly. Insert is a pie chart of the contribution of top 9 loci to total piRNAs in testis.

(D) Same as in (C) but for ovary.

(E) UCSC genome browser view of a peri-centromeric region (chrX) encompassing the entire flamenco locus (purple) and the distal part of $A T$-chX piRNA cluster (green). Below genomic coordinates (dm6) are piRNA coverage tracks using different read-inclusion criteria as defined in (A). Note that, whereas flamenco produce piRNAs that can be mostly mapped to unique genomic positions, $A T$-chX generates piRNAs that map to local repeats in this cluster, but nowhere else in the genome. Addition of non-local multi-mapper reads does not change the profile, indicating that the vast majority of piRNAs produced by the cluster are captured by unique+local mappings.

(F) UCSC genome browser view of the entire Y chromosome that harbors two Su(Ste) loci (blue) and the novel $h 17$ piRNA cluster (orange). piRNA coverage tracks using different read-inclusion criteria are shown below genomic coordinates (dm6). At the bottom, all known Y-linked proteinencoding genes are drawn for reference (not to exact scale). Note that piRNA profiles of Su(Ste) and $h 17$ clusters collapse if piRNAs derived from local repeats are excluded.

Figure 5. piRNA clusters are differentially employed to tame sex-specific TE expression.

(A) Plot showing the cumulative contribution of top piRNA clusters to the total piRNA populations in testis (left) and ovary (right), up to 100 clusters.

(B) Heatmaps showing piRNA production from major piRNA clusters. Note that $S u(S t e)$ and $h 17$ clusters are $\mathrm{Y}$-linked so there are no piRNAs from these loci in females that lack $\mathrm{Y}$ chromosome. (C) Bar graphs displaying the sex bias of piRNA cluster expression (left) and cumulative sex bias of the TE context for each cluster (right). Sex bias of piRNA cluster expression is defined as the $\log 2$ ratio of piRNA cluster expression in ovary over testis shown in (B), so ovary-biased ones are 
positive in value. Cumulative sex bias of cluster TE content is calculated by summing the sex bias of TEs (as described for Figure 2E) weighted by their length contributions to the cluster (equation shown on the right). An example is shown on the bottom right for a hypothetical cluster composed of two TEs with lengths and sex biases labeled accordingly for illustration. Only TEs showing strong sex biases were used in calculation. See also methods.

(D) TE composition of ovary-biased $42 A B$ cluster. Shown are fractions of $42 A B$ cluster occupied by sequences from top 6 TE families. These 6 TEs are completely absent in 38C, a testis-biased piRNA cluster. Expression of these 6 TEs is all ovary-biased (Figure S3A).

(E) Contributions of 3 testis-biased TEs (Figure S3A) to the ovary-biased $42 A B$ cluster and testisbiased $38 \mathrm{C}$ cluster. These TEs were selected as the most enriched by length in $38 \mathrm{C}$ compared to $42 A B$.

(F) The Sox102F gene generates piRNAs in ovary, but not in testis. This locus harbors a single autonomous TE, Tc1-2, that has ovary-biased expression (Figure S3A). piRNA coverage tracks show both uniquely-mapped and local repeat-derived reads.

\section{Figure 6. Hsp70B and h17 piRNA clusters encode piRNAs that target host genes.}

(A) Hsp70B piRNA cluster (top) and the putative target, nod (bottom). piRNA coverage tracks using different read-inclusion criteria are shown below RefSeq and genomic coordinates (dm6) for $H s p 70 B$ cluster. $\sim 354 \mathrm{bp}$ local repeats homologous to a $320 \mathrm{bp}$ exonic region of nod are depicted as solid blocks, which fill up most inter-TE space at this locus. Note that "unique+local" piRNA track does not include TE-derived piRNAs that map outside this locus, but it picks up bona fide local repeats that are homologous, but not identical, to nod.

(B) $h 17$ piRNA cluster on $Y$ chromosome. piRNA coverage tracks using different read-inclusion criteria are shown. Sequences with high levels of sequence similarity to protein-encoding genes are depicted as colored blocks (not to exact scale): CG12717 (green), Paics (orange), ProtA (blue). Note that gene-homologous islands fill up most inter-TE space at this locus. Genomic coordinates are based on dm6 genome assembly.

(C) Coverage of sense (unique, 0 mismatch) and antisense piRNAs (with up to 3 mismatches) over four putative, protein-encoding gene targets of testis piRNAs. Antisense piRNA abundance is shown for each gene. 
Figure 7. Regulation of CG12717/pita by small RNA pathways.

(A) MA plots showing gene expression changes from polyA+RNA-seq of aub (top), zuc (middle) and spn-E (bottom) mutant testes versus heterozygous sibling controls. Genes are marked red when passing a stringent statistical cutoff (adjusted $P<0.001$, from DESeq2). Additional coloring includes: CG12717/pita (green), annotated Stellate transcripts (orange), frtz (purple), and the mutated gene in each mutant (blue).

(B) Confocal images of pita mRNAs detected by in situ HCR in aub (top), zuc (middle) and spn-E (bottom) mutant testes along with respective heterozygous sibling controls. Probes were designed against a $\sim 400$ bp sequence unique to pita and absent on $Y$ (Figure S5B), so they do not target h17 piRNA precursors. Note that de-repression of pita in piRNA pathway mutants is observed specifically in differentiating spermatocytes (pointed to by orange arrows). Scale bar: $20 \mu \mathrm{m}$.

(C) Heatmaps showing fold-change of five protein-encoding genes in three mutant testes according to polyA+ RNA-seq shown in (A).

(D) Bar graphs displaying modENCODE data of pita and its paralog velo expression in $D$. melanogaster gonads of both sexes.

(E) Analysis of ping-pong processing of pita-mapping piRNAs. Histogram shows distribution of 5'to-5' distances of complementary piRNA pairs with an enrichment for 10nt (i.e., ping-pong signature). To select secondary piRNAs processed from pita transcripts, only reads that map perfectly to pita mRNAs in sense orientation and do not map perfectly to $h 17$ cluster were used in this analysis. Antisense piRNAs were selected allowing up to 3 mismatches.

(F) Cladogram of major species in Drosophila genus (left) and the evolutionary history of velo, pita and pita-related sequences in genomes of these species (right). Orthologs were identified based on sequence homology and synteny. Shown in purple are locations of additional pita copies in each species and copy numbers in parenthesis.

(G) Cartoon depicting distribution of pita-homologous sequences in D. mauritiana genome. Orthologous pita is marked blue, orthologous velo is marked green, and the duplicated, candidate sources of pita-targeting endo-siRNAs are marked red. Note that they scatter across pericentromeric heterochromatin of chrX and chr3, as well as chrY and scaffolds (not shown).

$(H)$ Profiles of pita-mapping small RNAs in testes of $D$. melanogaster (top) and D. mauritiana (bottom). Size distributions are shown on the left. Coverage plots over the orthologous pita gene in each species are shown on the right, including: cumulative alignment of heterochromatic, 
duplicated copies of pita over the syntenic, orthologous pita (top, solid bar), stranded coverage of 23-29nt piRNAs (middle, histogram) and 19-22nt endo-siRNAs (bottom, histogram) over the orthologous pita gene.

(I) Illustration showing two representative head-to-head copies of pita homology (red) in the pericentromeric heterochromatin of $D$. mauritiana $X$ chromosome. pita-related sequences are flanked by TEs and are part of a large inverted repeat that could potentially permit hpRNA biogenesis. 


\section{SUPPLEMENTARY ITEM TITLES AND LEGENDS}

Figure S1. Analysis pipeline of gonad small RNAs. Related to Figure 1.

Flow chart showing step-wise isolation of piRNAs from total small RNAs and subsequent mappings to different annotations (repeats, protein-encoding genes and genome).

Figure S2. Coverage of piRNAs over consensus sequences of complex satellites and examples of complementary Rsp-mapping piRNA pairs in two sexes. Related to Figure 1.

(A) Coverage plots of piRNAs over Rsp (top), HETRP (middle) and SAR (bottom), in testis (left) and ovary (right).

(B) Examples of complementary pairs of Rsp-mapping piRNAs. Note that in ovary (red) they show an enrichment for 10-nt overlap, i.e., ping-pong signature, but in testis (blue) they show near perfect-complementarity with no evidence for ping-pong signature.

Figure S3. TE levels in piRNA pathway mutants and curation of TEs regulated by Rhi in at least one sex. Related to Figure 2,3.

(A) Bar graphs showing TE levels in piRNA pathway mutant (rhi) testes (orange) and ovaries (blue). TEs that have at least 25 RPM in either sex is shown at the top, with the rest at the bottom.

(B) Table reporting manual curation of 36 confidently affected TE families by rhi $^{--}$. Silencing potential is TRUE when there are normally $>100$ RPM antisense piRNAs and they show $>2$-fold reduction in rhi mutants. TEs are deemed de-repressed when having $>3$-fold up-regulation. Note a few unexpected cases where TE de-repression is not accompanied by piRNA loss, the ovary ones of which were described before (Klattenhoff et al., 2009).

Figure S4. An algorithm that includes local repeats in piRNA cluster definition and analysis. Related to Figure 4.

(A) Flow chart showing steps of the new algorithm that includes local repeats in piRNA cluster definition and analysis. See also methods.

(B) Histogram showing the distribution of "max distances" defined in (A) to identify a meaningful cutoff $(2 \mathrm{Mb})$ for distinguishing local from non-local repeats. See also methods. 
Figure S5. Characterization of pita homology in D. mel. Related to Figure 6,7.

(A) Homology between two D. melanogaster paralogs: velo and CG12717/pita. The homologous regions are marked using BLAT and they share $75 \%$ nucleotide sequence identity.

(B) Alignment of duplicated, partial copies of CG12717 at $h 17$ on D. melanogaster $Y$ to its CG12717 gene (left), and their genomic coordinates (right). Note that there are two small regions of CG12717 absent on Y. RNA in situ HCR was targeted against the ORF region unique to CG12717 gene.

Table S1. Genome-wide piRNA clusters in testis and ovary as well as major piRNA clusters defined in this study. 


\section{MATERIALS AND METHODS}

\section{Fly stocks}

Stocks and crosses were raised at $25^{\circ} \mathrm{C}$. The following stocks were used: aub ${ }^{\text {QC42 }}$ (BDSC4968), $a u b^{H N 2}$ (BDSC8517), zuc ${ }^{D f}$ (BSDC3079), spn- $E^{\text {h/s3987 }}$ (BDSC24853) and spn- $E^{1}$ (BDSC3327) were obtained from Bloomington Drosophila Stock Center; $r h i^{2}$ and $r h i^{K G}$ were gifts of William Theurkauf; $z_{u C}{ }^{H M 27}$ was a gift from Trudi Schüpbach; nosP-GFP-Burdock was a gift from Julius Brennecke. Heterozygous siblings were used as controls for all experiments.

\section{RNA in situ hybridization chain reaction (HCR)}

A kit containing a DNA probe set, a DNA probe amplifier and hybridization, amplification and wash buffers were purchased from Molecular Instruments (molecularinstruments.org) for CG12717 transcripts. To avoid targeting the $h 17$ region on $Y$, we designed probes against a $\sim 400 \mathrm{bp}$ unique region present in CG12717 on $X$ but absent on $Y$ chromosome. The CG12717 probe set (unique identifier: 3916/E064) initiated B3 (Alexa546) amplifier. In situ HCR v3.0 (Choi et al., 2018) was performed according to manufacturer's recommendations for generic samples in solution.

\section{Image acquisition and analysis}

Confocal images were acquired with Zeiss LSM 800 using a 63x oil immersion objective (NA=1.4) and processed using Fiji (Schindelin et al., 2012). Single focal planes were shown in all images, where dotted outlines were drawn for illustration purposes.

\section{RNA-seq}

RNA was extracted from 160-200 pairs of dissected testes of aub $b^{Q C 42 / H N 2}, s p n-E^{1 / / h / s 3987}, z U c^{H M 27 / D f}$ and respective heterozygous sibling controls in TRIzol (Invitrogen). PolyA+ selection was done using NEBNext Poly(A) mRNA Magnetic Isolation Module (NEB E7490), followed by strandspecific library prep with NEBNext Ultra Directional RNA Library Prep Kit for Illumina (NEB E7760) according to manufacturer's instructions. Libraries were sequenced on Illumina HiSeq 2500 yielding 11-17 million 50bp single-end reads. PolyA-selected RNA-seq of rhi mutants and controls were downloaded from NCBI SRA (see the accompanying manuscript for testis and GSE126578 for ovary, 2 biological replicates per sex per genotype). 


\section{RNA-seq analysis}

To quantify expression levels of protein-encoding genes across different piRNA pathway mutants (aub, zuc and spn-E), we used kallisto 0.46.1 (Bray et al., 2016). Three heterozygous controls were pooled as triplicates of controls to be analyzed against duplicates of each of the three piRNA pathway mutants. Transcript-level quantification was pooled to obtain gene-level quantification. Differential gene expression was done with DESeq2 (Love et al., 2014). Expression of CG12717 and veloren in ovary and testis from modENCODE (Brown et al., 2014) was extracted from FlyBase (Thurmond et al., 2019).

For analysis of TE expression and TE fold-change in piRNA pathway mutants of both sexes, rhi mutants were used where piRNA production from germline-specific dual-strand clusters was abolished. Reads mapped to rRNA were discarded using bowtie 1.2.2 allowing 3 mismatches. Reads were then mapped to TE consensus from RepBase17.08 using bowtie 1.2.2 with -v 3 -k 1 and normalized to the total number of reads mapped to dm6 genome. For simplicity, reads mapped to LTR and internal sequences were merged for each LTR TE given their well correlative behaviors. Only TEs that have $\geq 5$ RPM expression in piRNA pathway mutants of either sex were kept for the analysis $(n=87)$. A pseudo-count of 1 was added before calculating TE fold-change in piRNA pathway mutants.

\section{Identification of TEs regulated by rhi}

To identify a set of TEs regulated by $r h i$ in at least one sex, we looked for TEs that have at least 100 RPM in rhi mutant ovaries or at least 25 RPM in rhi mutant testes. Next, we filter out TEs that show less than 3-fold de-repression in both sexes. From the initial 87 TEs defined above, these led to a total of 36 TEs regulated by $r h i$ in at least one sex shown in Figure 2E and Figure 3A. See Figure S3B for detailed profiles of these 36 TEs.

\section{piRNA-seq}

RNA extraction was done as above for RNA-seq. 18-30nt small RNAs were purified by PAGE (15\% polyacrylamide gel) from $\sim 1 \mu$ g total RNA. Purified small RNA was subject to library prep using NEBNext Multiplex Small RNA Sample Prep Set for Illumina (NEB E7330) according to manufacturer's instructions. Adaptor-ligated, reverse-transcribed, PCR-amplified samples were 
purified again by PAGE (6\% polyacrylamide gel). Two biological replicates per genotype were sequenced on Illumina HiSeq 2500 yielding 15-20 million 50bp single-end reads.

\section{piRNA-seq analysis of TEs, complex satellites and genes}

To isolate piRNAs, adaptor-trimmed total small RNAs were size-selected for 23-29nt (cutadapt 2.5) and those mapped to rRNA, miRNA, snRNA, snoRNA and tRNA were discarded (bowtie 1.2.2 with -v 3). piRNAs were first mapped to RepBase17.08 to obtain the portion mapping to TEs and complex satellites; the rest was then mapped to gene sequences derived from the gtf file downloaded from Ensembl (BDGP6.28.99) (Yates et al., 2019); reads unmapped to repeats and genes were then mapped to dm6 to infer the portion mapping to inter-genic regions, and the unmapped ones were listed under "others" category. A pipeline is also drawn in Figure S1. For TE-antisense piRNA analysis, piRNA reads were mapped, normalized and processed as done for polyA+ RNA-seq (see above). For complex satellite-mapping small RNAs, we plotted size distribution, analyzed nucleotide bias at position 1 and calculated coverage along consensus sequences using bedtools v2.28.0. Ping-pong signature analysis (i.e., 5'-to-5' distances between complementary piRNA pairs) was done with custom scripts. Ping-pong z-score was calculated using 1-9nt and 11-23nt as background distribution for an enrichment of 10nt. For piRNAs antisense to protein-encoding genes of interest, we downloaded gene sequences from FlyBase (Thurmond et al., 2019) and mapped piRNAs to them using bowtie 1.2.2. For mRNA-derived sense piRNAs, we mapped piRNAs to genome and kept ones with unique mapping and zero mismatch (bowtie 1.2.2 with $-\mathrm{v} 0-\mathrm{m} 1$ ) to the gene regions and orientations of interest.

\section{A pipeline tolerating local repeats for piRNA cluster analysis}

We first separated rRNA-depleted 23-29nt small RNA reads that map to one unique location in the genome and others that have multiple mapping positions ("multi-mappers"). For all multimappers, we filtered out those who map to more than one chromosome arm, retaining only ones with multi-mapping positions on a single chromosome arm ("intra-chromosomal repeats"). Then, for each of the reads we kept as intra-chromosomal repeats, we calculated the maximum distance ("max distance") of all mapping positions. In order to enforce the local requirement, we hoped to identify a cutoff distance for max distances, which is large enough to contain known piRNA loci but small enough to allow certain resolution of neighboring loci. To this end, we analyzed a pool of 50bp DNA fragments tiling the entire dm6 genome and plotted a histogram of max distances 
for all intra-chromosomal repeats (Figure S4B). This revealed a density of intra-chromosomal repeats having max distances smaller than $\sim 500 \mathrm{~Kb}$, as well as four pronounced peaks with larger max distances. Sequence analysis uncovered the identities of these peaks: the peak with $\sim 600 \mathrm{~Kb}$ max distance corresponds to $A T$-chX, the peak with $\sim 1.8 \mathrm{Mb}$ max distance represents $S u(S t e)$, and the other two peaks mostly contain $\mathrm{Y}$-specific simple repeats. We thus set a $2 \mathrm{Mb}$ tolerance threshold of max distances to allow local repeats in piRNA cluster analysis. In other words, we defined local repeats as repeats that have all copies contained within a window smaller than $2 \mathrm{Mb}$ and merged their normalized counts with unique sequences for piRNA cluster analysis. Alignment was done using bowtie 2 to dm6 genome. To compare this new pipeline with other standard approaches (permitting only unique mappers or allowing all multi-mappers with randomly assigned locations), we calculated the number of reads mapped to major piRNA clusters using different methods (Figure 4B). A summary of this pipeline is shown on Figure S4A.

\section{Definition of piRNA clusters}

23-29nt small RNAs were mapped to dm6 genome using the above-mentioned pipeline tolerating local repeats and generated coverage profiles across $1 \mathrm{~Kb}$ windows that tile the genome. $1 \mathrm{~Kb}$ windows including highly expressed miRNA, snRNA, snoRNA, hpRNA or 7SL SRP RNA were excluded. $1 \mathrm{~Kb}$ windows with low read-coverage ( $\leq 100 \mathrm{bp})$ were also excluded. Then, $1 \mathrm{~Kb}$ windows that produce at least certain amounts of piRNAs were extracted for cluster definition ( $\geq 10 R P M$ for testis, $\geq 50$ RPM for ovary). Neighboring $1 \mathrm{~Kb}$ widows within $3 \mathrm{~Kb}$ were merged. If merged windows were $\geq 5 \mathrm{~Kb}$, they were merged again within $15 \mathrm{~Kb}$. This yields 844 piRNA clusters in testis and 525 piRNA clusters in ovary, after manual curation. Major piRNA clusters described before in ovaries (Brennecke et al., 2007; Mohn et al., 2014) were all recovered with similar resolution. To compare expression levels of major piRNA clusters between sexes, cluster boundaries were manually curated to guarantee identical regions being compared. piRNA clusters defined in this study for both sexes are listed in Table S1.

\section{TE content of piRNA clusters}

TE annotation in dm6 genome was downloaded from UCSC Table Browser (Karolchik et al., 2004). piRNA cluster boundaries were defined as described above. For piRNA cluster of interest, the TE content is calculated as length contribution to the entire cluster length by individual TEs. TE contents add up to less than $100 \%$, as TEs do not fill completely the cluster length. 


\section{Sex bias of piRNA cluster TE content}

Sex bias of individual TEs was first computed as log2 ratio of expression levels in piRNA pathway mutants (rhi) between sex (ovary over testis). Sex bias of piRNA cluster TE content was then computed as the cumulative sex bias of individual TEs inside the cluster, weighted by their length contribution to the cluster. Using all expressed TEs or only ones that show pronounced sex bias generated comparable results. To eliminate noise, we only used TEs that exhibit strong, $\geq 10$-fold sexual difference in expression $(n=24)$. An equation and an example are shown in Figure $5 C$.

\section{BLAT and BLAST analysis}

To characterize the unannotated sequence between annotated repeats in piRNA clusters, interrepeat sequences were analyzed using BLAT on UCSC Genome browser (Kent, 2002). For example, an inter-TE sequence at $H s p 70 B$ locus was used to BLAT against dm6 genome, which revealed the homology with an exon of nod gene (Figure 6A). Homology between CG12717 and veloren was done with both BLAT and BLAST, which yielded similar results. Characterization of CG12717-homologous sequences at $h 17$ locus (Figure S5B) was done by multiple sequence alignment with the Needle program (ebi.ac.uk/Tools/psa/emboss_needle/).

\section{Phylogenetic analysis}

The longest transcripts of veloren and CG12717 in D. melanogaster genome were used to BLAST against nucleotide collection with blastN program. Orthologs of these two genes in other Drosophila species were identified based on high nucleotide similarity and synteny. In all orthologs identified for both genes, we found the same flanking protein-encoding genes, confirming their ortholog identities. Occasionally, BLAST with CG12717 revealed the veloren ortholog in that species as well; but only in $D$. mauritiana, $D$. simulans and $D$. sechellia genomes are there additional hits with high sequence homology to CG12717, other than the orthologous CG12717 and veloren. These additional CG12717-related sequences are in some cases annotated as predicted genes, but all buried in TE-rich heterochromatin (close to centromere or in highly repetitive unassigned scaffolds). To examine the organization of CG12717-related sequences in D. mauritiana genome in detail, we ran BLAST using D. mauritiana CG12717 gene against its genome (assembly: GCA_004382145.1), which revealed additional unannotated 
regions with high sequence similarity to CG12717. Those located on chrX and chr3 were drawn in Figure 7G. The instance where two adjacent CG12717-related sequences are arranged headto-head on chrX is illustrated in Figure 7I, and the other three such instances are found in unassigned scaffolds. To uncover the identity of flanking unannotated sequences, we BLAST the $50 \mathrm{~Kb}$ region encompassing CG12717-related sequences against TE consensus (RepBase17.08). The cladogram was drawn for illustration (Drosophila 12 Genomes Consortium, 2007).

\section{Analysis of testis small RNAs in non-D. melanogaster species}

Testis small RNA libraries from non- $D$. melanogaster species was downloaded from NCBI SRA: D. simulans SRR7410589 (Lin et al., 2018) and D. mauritiana SRR7961897 (Kotov et al., 2019). Adaptor-trimmed reads were mapped to the orthologous CG12717 gene, D. simulans GD15918 and D. mauritiana LOC117148327, respectively (bowtie 1.2.2 with $-\mathrm{v} 3-\mathrm{k} 1$ ). Coverage was plotted along the orthologous CG12717 gene.

\section{Data visualization and statistical analysis}

Most data visualization and statistical analysis were done in Python 3 via JupyterLab with the following software packages: numpy (Oliphant, 2015), pandas (McKinney, 2010) and altair (VanderPlas et al., 2018). The UCSC Genome Brower (Kent et al., 2002) and IGV (Robinson et al., 2011; Thorvaldsdóttir et al., 2013) were used to explore sequencing data and to prepare browser track panels shown.

\section{Data and code availability}

Sequencing data will be uploaded to NCBI SRA. 


\section{REFERENCES}

Aravin, A.A. (2020). Pachytene piRNAs as beneficial regulators or a defense system gone rogue. Nat. Genet. 52, 644-645.

Aravin, A., Gaidatzis, D., Pfeffer, S., Lagos-Quintana, M., Landgraf, P., lovino, N., Morris, P., Brownstein, M.J., Kuramochi-Miyagawa, S., Nakano, T., et al. (2006). A novel class of small RNAs bind to MILI protein in mouse testes. Nature 442, 203-207.

Aravin, A.A., Naumova, N.M., Tulin, A.V., Vagin, V.V., Rozovsky, Y.M., and Gvozdev, V.A. (2001). Double-stranded RNA-mediated silencing of genomic tandem repeats and transposable elements in the D. melanogaster germline. Current Biology 11, 1017-1027.

Aravin, A.A., Klenov, M.S., Vagin, V.V., Bantignies, F., Cavalli, G., and Gvozdev, V.A. (2004). Dissection of a Natural RNA Silencing Process in the Drosophila melanogaster Germ Line. MCB 24, 6742-6750.

Beall, E.L., Lewis, P.W., Bell, M., Rocha, M., Jones, D.L., and Botchan, M.R. (2007). Discovery of tMAC: a Drosophila testis-specific meiotic arrest complex paralogous to Myb-Muv B. Genes Dev. 21, 904-919.

Berdnik, D., Favaloro, V., and Luo, L. (2012). The SUMO protease Verloren regulates dendrite and axon targeting in olfactory projection neurons. J. Neurosci. 32, 8331-8340.

Bernardo Carvalho, A., Koerich, L.B., and Clark, A.G. (2009). Origin and evolution of Y chromosomes: Drosophila tales. Trends in Genetics 25, 270-277.

Bray, N.L., Pimentel, H., Melsted, P., and Pachter, L. (2016). Near-optimal probabilistic RNAseq quantification. Nat Biotechnol 34, 525-527.

Brennecke, J., Aravin, A.A., Stark, A., Dus, M., Kellis, M., Sachidanandam, R., and Hannon, G.J. (2007). Discrete Small RNA-Generating Loci as Master Regulators of Transposon Activity in Drosophila. Cell 128, 1089-1103.

Brown, J.B., Boley, N., Eisman, R., May, G.E., Stoiber, M.H., Duff, M.O., Booth, B.W., Wen, J., Park, S., Suzuki, A.M., et al. (2014). Diversity and dynamics of the Drosophila transcriptome. Nature 512, 393-399.

Carmell, M.A., Girard, A., van de Kant, H.J.G., Bourc'his, D., Bestor, T.H., de Rooij, D.G., and Hannon, G.J. (2007). MIWI2 is essential for spermatogenesis and repression of transposons in the mouse male germline. Dev. Cell 12, 503-514.

Carpenter, A.T. (1973). A meiotic mutant defective in distributive disjunction in Drosophila melanogaster. Genetics 73, 393-428.

Carvalho, A.B., Vicoso, B., Russo, C.A.M., Swenor, B., and Clark, A.G. (2015). Birth of a new gene on the $Y$ chromosome of Drosophila melanogaster. Proc Natl Acad Sci USA 112, 12450 12455.

Choi, H.M.T., Schwarzkopf, M., Fornace, M.E., Acharya, A., Artavanis, G., Stegmaier, J., Cunha, A., and Pierce, N.A. (2018). Third-generation in situ hybridization chain reaction: multiplexed, quantitative, sensitive, versatile, robust. Development 145. 
Czech, B., Malone, C.D., Zhou, R., Stark, A., Schlingeheyde, C., Dus, M., Perrimon, N., Kellis, M., Wohlschlegel, J.A., Sachidanandam, R., et al. (2008). An endogenous small interfering RNA pathway in Drosophila. Nature 453, 798-802.

Deng, W., and Lin, H. (2002). miwi, a murine homolog of piwi, encodes a cytoplasmic protein essential for spermatogenesis. Dev. Cell 2, 819-830.

Drosophila 12 Genomes Consortium (2007). Evolution of genes and genomes on the Drosophila phylogeny. Nature 450, 203-218.

EIMaghraby, M.F., Andersen, P.R., Pühringer, F., Hohmann, U., Meixner, K., Lendl, T., Tirian, L., and Brennecke, J. (2019). A Heterochromatin-Specific RNA Export Pathway Facilitates piRNA Production. Cell 178, 964-979.e20.

Gatti, M., and Pimpinelli, S. (1983). Cytological and genetic analysis of the $Y$ chromosome of Drosophila melanogaster: I. Organization of the fertility factors. Chromosoma 88, 349-373.

Gell, S.L., and Reenan, R.A. (2013). Mutations to the piRNA Pathway Component Aubergine Enhance Meiotic Drive of Segregation Distorter in Drosophila melanogaster. Genetics 193, 771784.

Girard, A., Sachidanandam, R., Hannon, G.J., and Carmell, M.A. (2006). A germline-specific class of small RNAs binds mammalian Piwi proteins. Nature 442, 199-202.

Gonzalez, J., Qi, H., Liu, N., and Lin, H. (2015). Piwi Is a Key Regulator of Both Somatic and Germline Stem Cells in the Drosophila Testis. Cell Reports 12, 150-161.

Hall, I.M., Shankaranarayana, G.D., Noma, K.-I., Ayoub, N., Cohen, A., and Grewal, S.I.S. (2002). Establishment and maintenance of a heterochromatin domain. Science 297, 22322237.

Handler, D., Meixner, K., Pizka, M., Lauss, K., Schmied, C., Gruber, F.S., and Brennecke, J. (2013). The Genetic Makeup of the Drosophila piRNA Pathway. Molecular Cell 50, 762-777.

Hartl, D.L. (1973). Complementation analysis of male fertility among the segregation distorter chromosomes of Drosophila melanogaster. Genetics 73, 613-629.

Hawley, R.S., and Theurkauf, W.E. (1993). Requiem for distributive segregation: achiasmate segregation in Drosophila females. Trends Genet. 9, 310-317.

Hiller, M., Chen, X., Pringle, M.J., Suchorolski, M., Sancak, Y., Viswanathan, S., Bolival, B., Lin, T.-Y., Marino, S., and Fuller, M.T. (2004). Testis-specific TAF homologs collaborate to control a tissue-specific transcription program. Development 131, 5297-5308.

Houwing, S., Kamminga, L.M., Berezikov, E., Cronembold, D., Girard, A., van den Elst, H., Filippov, D.V., Blaser, H., Raz, E., Moens, C.B., et al. (2007). A Role for Piwi and piRNAs in Germ Cell Maintenance and Transposon Silencing in Zebrafish. Cell 129, 69-82.

Houwing, S., Berezikov, E., and Ketting, R.F. (2008). Zili is required for germ cell differentiation and meiosis in zebrafish. EMBO J 27, 2702-2711. 
Hsieh, T., and Brutlag, D. (1979). Sequence and sequence variation within the $1.688 \mathrm{~g} / \mathrm{cm} 3$ satellite DNA of Drosophila melanogaster. J. Mol. Biol. 135, 465-481.

Kamminga, L.M., Luteijn, M.J., den Broeder, M.J., Redl, S., Kaaij, L.J.T., Roovers, E.F., Ladurner, P., Berezikov, E., and Ketting, R.F. (2010). Hen1 is required for oocyte development and piRNA stability in zebrafish. EMBO J 29, 3688-3700.

Kano, H., Godoy, I., Courtney, C., Vetter, M.R., Gerton, G.L., Ostertag, E.M., and Kazazian, H.H. (2009). L1 retrotransposition occurs mainly in embryogenesis and creates somatic mosaicism. Genes Dev. 23, 1303-1312.

Karolchik, D., Hinrichs, A.S., Furey, T.S., Roskin, K.M., Sugnet, C.W., Haussler, D., and Kent, W.J. (2004). The UCSC Table Browser data retrieval tool. Nucleic Acids Res. 32, D493-496.

Karpen, G.H., and Spradling, A.C. (1992). Analysis of subtelomeric heterochromatin in the Drosophila minichromosome Dp1187 by single P element insertional mutagenesis. Genetics 132, 737-753.

Kent, W.J. (2002). BLAT--the BLAST-like alignment tool. Genome Res. 12, 656-664.

Kent, W.J., Sugnet, C.W., Furey, T.S., Roskin, K.M., Pringle, T.H., Zahler, A.M., and Haussler, D. (2002). The human genome browser at UCSC. Genome Res. 12, 996-1006.

Khost, D.E., Eickbush, D.G., and Larracuente, A.M. (2017). Single-molecule sequencing resolves the detailed structure of complex satellite DNA loci in Drosophila melanogaster. Genome Res. 27, 709-721.

Klattenhoff, C., Xi, H., Li, C., Lee, S., Xu, J., Khurana, J.S., Zhang, F., Schultz, N., Koppetsch, B.S., Nowosielska, A., et al. (2009). The Drosophila HP1 Homolog Rhino Is Required for Transposon Silencing and piRNA Production by Dual-Strand Clusters. Cell 138, 1137-1149.

Klein, J.D., Qu, C., Yang, X., Fan, Y., Tang, C., and Peng, J.C. (2016). c-Fos Repression by Piwi Regulates Drosophila Ovarian Germline Formation and Tissue Morphogenesis. PLoS Genet 12, e1006281.

Kofler, R., Nolte, V., and Schlötterer, C. (2015). Tempo and Mode of Transposable Element Activity in Drosophila. PLoS Genet. 11, e1005406.

Kotov, A.A., Adashev, V.E., Godneeva, B.K., Ninova, M., Shatskikh, A.S., Bazylev, S.S., Aravin, A.A., and Olenina, L.V. (2019). piRNA silencing contributes to interspecies hybrid sterility and reproductive isolation in Drosophila melanogaster. Nucleic Acids Research 47, 4255-4271.

Krsticevic, F.J., Santos, H.L., Januário, S., Schrago, C.G., and Carvalho, A.B. (2010). Functional Copies of the Mst77F Gene on the Y Chromosome of Drosophila melanogaster. Genetics 184, 295-307.

Kuramochi-Miyagawa, S., Kimura, T., ljiri, T.W., Isobe, T., Asada, N., Fujita, Y., Ikawa, M., Iwai, N., Okabe, M., Deng, W., et al. (2004). Mili, a mammalian member of piwi family gene, is essential for spermatogenesis. Development 131, 839-849. 
Larracuente, A.M., and Presgraves, D.C. (2012). The Selfish Segregation Distorter Gene Complex of Drosophila melanogaster. Genetics 192, 33-53.

Le Thomas, A., Rogers, A.K., Webster, A., Marinov, G.K., Liao, S.E., Perkins, E.M., Hur, J.K., Aravin, A.A., and Tóth, K.F. (2013). Piwi induces piRNA-guided transcriptional silencing and establishment of a repressive chromatin state. Genes Dev. 27, 390-399.

Le Thomas, A., Stuwe, E., Li, S., Du, J., Marinov, G., Rozhkov, N., Chen, Y.-C.A., Luo, Y., Sachidanandam, R., Toth, K.F., et al. (2014). Transgenerationally inherited piRNAs trigger piRNA biogenesis by changing the chromatin of piRNA clusters and inducing precursor processing. Genes Dev. 28, 1667-1680.

Lerat, E., Burlet, N., Biémont, C., and Vieira, C. (2011). Comparative analysis of transposable elements in the melanogaster subgroup sequenced genomes. Gene 473, 100-109.

Li, C., Vagin, V.V., Lee, S., Xu, J., Ma, S., Xi, H., Seitz, H., Horwich, M.D., Syrzycka, M., Honda, B.M., et al. (2009). Collapse of Germline piRNAs in the Absence of Argonaute3 Reveals Somatic piRNAs in Flies. Cell 137, 509-521.

Lin, H., and Spradling, A.C. (1997). A novel group of pumilio mutations affects the asymmetric division of germline stem cells in the Drosophila ovary. Development 124, 2463-2476.

Lin, C.-J., Hu, F., Dubruille, R., Vedanayagam, J., Wen, J., Smibert, P., Loppin, B., and Lai, E.C. (2018). The hpRNA/RNAi Pathway Is Essential to Resolve Intragenomic Conflict in the Drosophila Male Germline. Developmental Cell 46, 316-326.e5.

Lohe, A.R., Hilliker, A.J., and Roberts, P.A. (1993). Mapping simple repeated DNA sequences in heterochromatin of Drosophila melanogaster. Trends in Genetics 9, 379.

Love, M.I., Huber, W., and Anders, S. (2014). Moderated estimation of fold change and dispersion for RNA-seq data with DESeq2. Genome Biol 15, 550.

Malone, C.D., Brennecke, J., Dus, M., Stark, A., McCombie, W.R., Sachidanandam, R., and Hannon, G.J. (2009). Specialized piRNA Pathways Act in Germline and Somatic Tissues of the Drosophila Ovary. Cell 137, 522-535.

McKinney, W. (2010). Data Structures for Statistical Computing in Python. (Austin, Texas), pp. 56-61.

Mendez-Lago, M., Bergman, C.M., de Pablos, B., Tracey, A., Whitehead, S.L., and Villasante, A. (2011). A Large Palindrome With Interchromosomal Gene Duplications in the Pericentromeric Region of the D. melanogaster Y Chromosome. Molecular Biology and Evolution 28, 19671971.

Mills, W.K., Lee, Y.C.G., Kochendoerfer, A.M., Dunleavy, E.M., and Karpen, G.H. (2019). RNA from a simple-tandem repeat is required for sperm maturation and male fertility in Drosophila melanogaster. ELife 8, e48940.

Mohn, F., Sienski, G., Handler, D., and Brennecke, J. (2014). The Rhino-Deadlock-Cutoff Complex Licenses Noncanonical Transcription of Dual-Strand piRNA Clusters in Drosophila. Cell 157, 1364-1379. 
Nishida, K.M., Saito, K., Mori, T., Kawamura, Y., Nagami-Okada, T., Inagaki, S., Siomi, H., and Siomi, M.C. (2007). Gene silencing mechanisms mediated by Aubergine piRNA complexes in Drosophila male gonad. RNA 13, 1911-1922.

Oliphant, T.E. (2015). Guide to NumPy (Austin, Tex.: Continuum Press).

Ozata, D.M., Gainetdinov, I., Zoch, A., O'Carroll, D., and Zamore, P.D. (2019). PIWI-interacting RNAs: small RNAs with big functions. Nat Rev Genet 20, 89-108.

Özata, D.M., Yu, T., Mou, H., Gainetdinov, I., Colpan, C., Cecchini, K., Kaymaz, Y., Wu, P.-H., Fan, K., Kucukural, A., et al. (2020). Evolutionarily conserved pachytene piRNA loci are highly divergent among modern humans. Nat Ecol Evol 4, 156-168.

Pane, A., Wehr, K., and Schüpbach, T. (2007). zucchini and squash Encode Two Putative Nucleases Required for rasiRNA Production in the Drosophila Germline. Developmental Cell 12, 851-862.

Robinson, J.T., Thorvaldsdóttir, H., Winckler, W., Guttman, M., Lander, E.S., Getz, G., and Mesirov, J.P. (2011). Integrative genomics viewer. Nat Biotechnol 29, 24-26.

Rojas-Ríos, P., and Simonelig, M. (2018). piRNAs and PIWI proteins: regulators of gene expression in development and stem cells. Development 145, dev161786.

Rojas-Ríos, P., Chartier, A., Pierson, S., and Simonelig, M. (2017). Aubergine and pi RNA s promote germline stem cell self-renewal by repressing the proto-oncogene Cbl. EMBO J 36, 3194-3211.

Rozhkov, N.V., Aravin, A.A., Zelentsova, E.S., Schostak, N.G., Sachidanandam, R., McCombie, W.R., Hannon, G.J., and Evgen'ev, M.B. (2010). Small RNA-based silencing strategies for transposons in the process of invading Drosophila species. RNA 16, 1634-1645.

Rozhkov, N.V., Hammell, M., and Hannon, G.J. (2013). Multiple roles for Piwi in silencing Drosophila transposons. Genes Dev. 27, 400-412.

Saint-Leandre, B., Capy, P., Hua-Van, A., and Filée, J. (2020). piRNA and Transposon Dynamics in Drosophila: A Female Story. Genome Biology and Evolution 12, 931-947.

Saito, K., Nishida, K.M., Mori, T., Kawamura, Y., Miyoshi, K., Nagami, T., Siomi, H., and Siomi, M.C. (2006). Specific association of Piwi with rasiRNAs derived from retrotransposon and heterochromatic regions in the Drosophila genome. Genes Dev. 20, 2214-2222.

Saito, K., Inagaki, S., Mituyama, T., Kawamura, Y., Ono, Y., Sakota, E., Kotani, H., Asai, K., Siomi, H., and Siomi, M.C. (2009). A regulatory circuit for piwi by the large Maf gene traffic jam in Drosophila. Nature 461, 1296-1299.

Sandler, L., Hiraizumi, Y., and Sandler, I. (1959). Meiotic Drive in Natural Populations of Drosophila Melanogaster. I. the Cytogenetic Basis of Segregation-Distortion. Genetics 44, 233250. 
Schindelin, J., Arganda-Carreras, I., Frise, E., Kaynig, V., Longair, M., Pietzsch, T., Preibisch, S., Rueden, C., Saalfeld, S., Schmid, B., et al. (2012). Fiji: an open-source platform for biological-image analysis. Nat. Methods 9, 676-682.

Schmidt, A., Palumbo, G., Bozzetti, M.P., Tritto, P., Pimpinelli, S., and Schäfer, U. (1999). Genetic and molecular characterization of sting, a gene involved in crystal formation and meiotic drive in the male germ line of Drosophila melanogaster. Genetics 151, 749-760.

Sienski, G., Dönertas, D., and Brennecke, J. (2012). Transcriptional silencing of transposons by Piwi and maelstrom and its impact on chromatin state and gene expression. Cell 151, 964-980.

Stapleton, W., Das, S., and McKee, B.D. (2001). A role of the Drosophila homeless gene in repression of Stellate in male meiosis. Chromosoma 110, 228-240.

Thorvaldsdóttir, H., Robinson, J.T., and Mesirov, J.P. (2013). Integrative Genomics Viewer (IGV): high-performance genomics data visualization and exploration. Brief. Bioinformatics 14, 178-192.

Thurmond, J., Goodman, J.L., Strelets, V.B., Attrill, H., Gramates, L.S., Marygold, S.J., Matthews, B.B., Millburn, G., Antonazzo, G., Trovisco, V., et al. (2019). FlyBase 2.0: the next generation. Nucleic Acids Research 47, D759-D765.

Vagin, V.V., Sigova, A., Li, C., Seitz, H., Gvozdev, V., and Zamore, P.D. (2006). A distinct small RNA pathway silences selfish genetic elements in the germline. Science 313, 320-324.

VanderPlas, J., Granger, B., Heer, J., Moritz, D., Wongsuphasawat, K., Satyanarayan, A., Lees, E., Timofeev, I., Welsh, B., and Sievert, S. (2018). Altair: Interactive Statistical Visualizations for Python. JOSS 3, 1057.

Volpe, T.A., Kidner, C., Hall, I.M., Teng, G., Grewal, S.I.S., and Martienssen, R.A. (2002). Regulation of heterochromatic silencing and histone H3 lysine- 9 methylation by RNAi. Science 297, 1833-1837.

Wang, S.H., and Elgin, S.C.R. (2011). Drosophila Piwi functions downstream of piRNA production mediating a chromatin-based transposon silencing mechanism in female germ line. Proc. Natl. Acad. Sci. U.S.A. 108, 21164-21169.

Wen, J., Duan, H., Bejarano, F., Okamura, K., Fabian, L., Brill, J.A., Bortolamiol-Becet, D., Martin, R., Ruby, J.G., and Lai, E.C. (2015). Adaptive Regulation of Testis Gene Expression and Control of Male Fertility by the Drosophila Hairpin RNA Pathway. Molecular Cell 57, 165178.

Wu, C.I., Lyttle, T.W., Wu, M.L., and Lin, G.F. (1988). Association between a satellite DNA sequence and the Responder of Segregation Distorter in D. melanogaster. Cell 54, 179-189.

Wu, P.-H., Fu, Y., Cecchini, K., Özata, D.M., Arif, A., Yu, T., Colpan, C., Gainetdinov, I., Weng, Z., and Zamore, P.D. (2020). The evolutionarily conserved piRNA-producing locus pi6 is required for male mouse fertility. Nat Genet 52, 728-739. 
Yates, A.D., Achuthan, P., Akanni, W., Allen, J., Allen, J., Alvarez-Jarreta, J., Amode, M.R., Armean, I.M., Azov, A.G., Bennett, R., et al. (2019). Ensembl 2020. Nucleic Acids Research gkz966.

Zhang, P., Knowles, B.A., Goldstein, L.S., and Hawley, R.S. (1990). A kinesin-like protein required for distributive chromosome segregation in Drosophila. Cell 62, 1053-1062.

Zhang, Z., Wang, J., Schultz, N., Zhang, F., Parhad, S.S., Tu, S., Vreven, T., Zamore, P.D., Weng, Z., and Theurkauf, W.E. (2014). The HP1 Homolog Rhino Anchors a Nuclear Complex that Suppresses piRNA Precursor Splicing. Cell 157, 1353-1363. 


\section{Figure 1. Chen et al.}

A

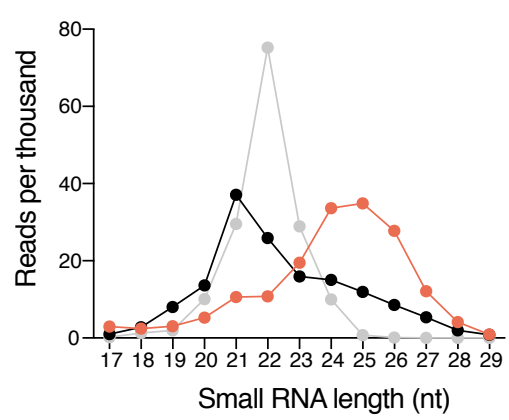

B

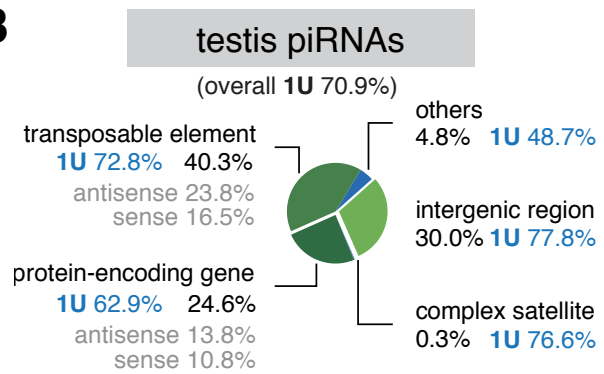

C
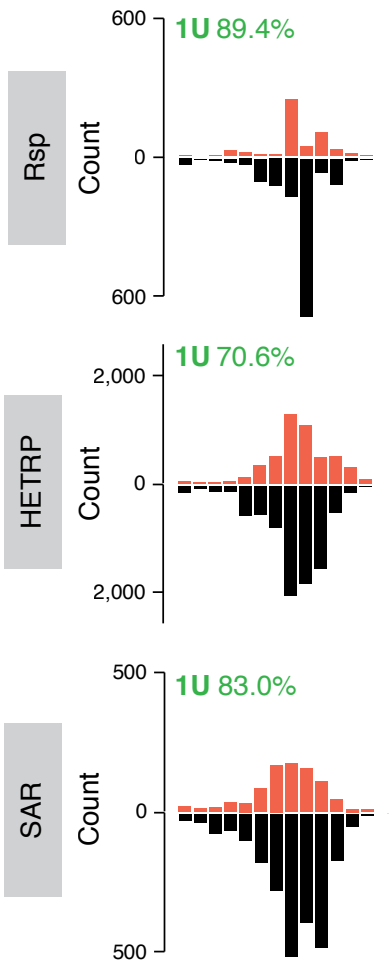

182022242628 Small RNA length (nt)

testis
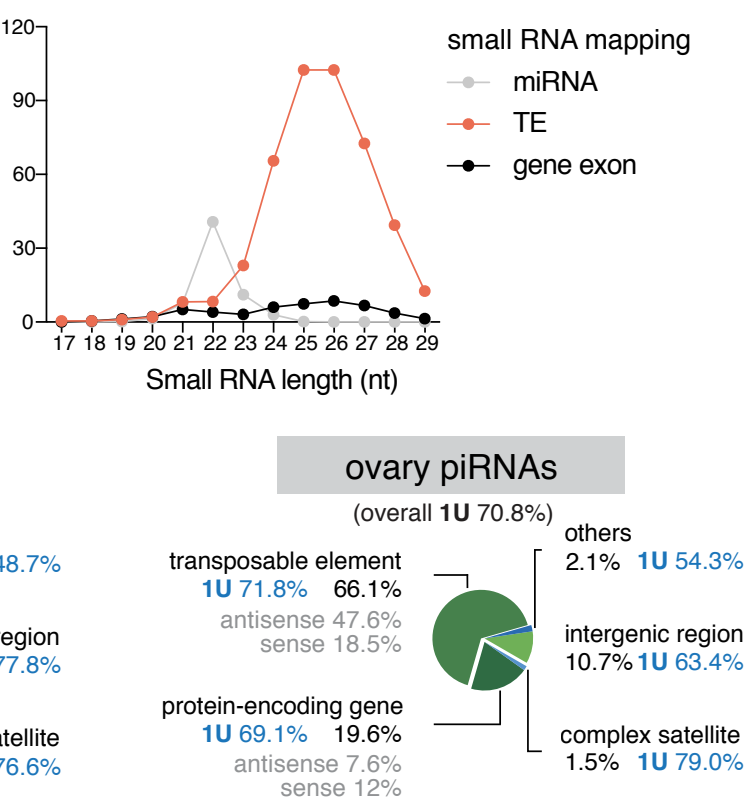

ovary

size distribution ping-pong signature
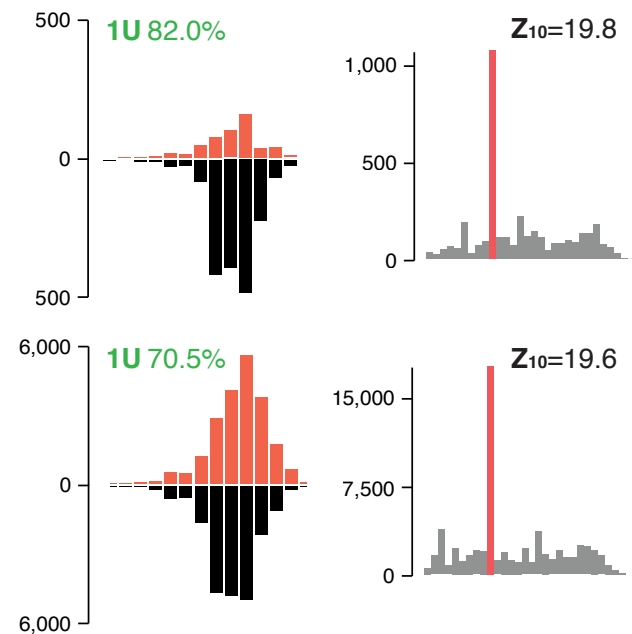

6,000

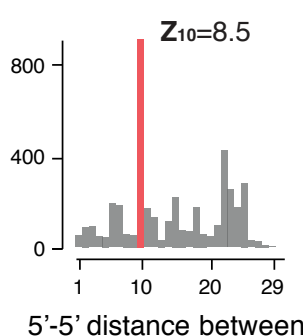

5'-5' distance between piRNA pairs (nt)
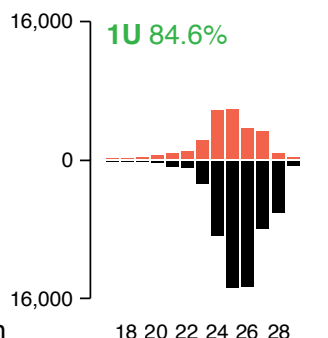

182022242628 Small RNA length (nt)

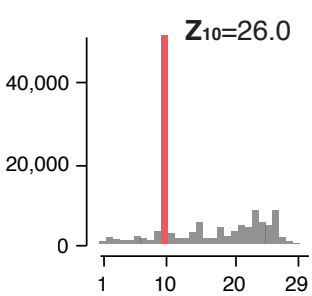

5'-5' distance between piRNA pairs (nt) 
bioRxiv preprint doi: https://doi.org/10.1101/2020.08.25.266585; this version posted August 25, 2020. The copyright holder for this preprint (which was not certified by peer review) is the author/funder, who has granted bioRxiv a license to display the preprint in perpetuity. It is made available under aCC-BY-NC-ND 4.0 International license.

Figure 2. Chen et al.

A

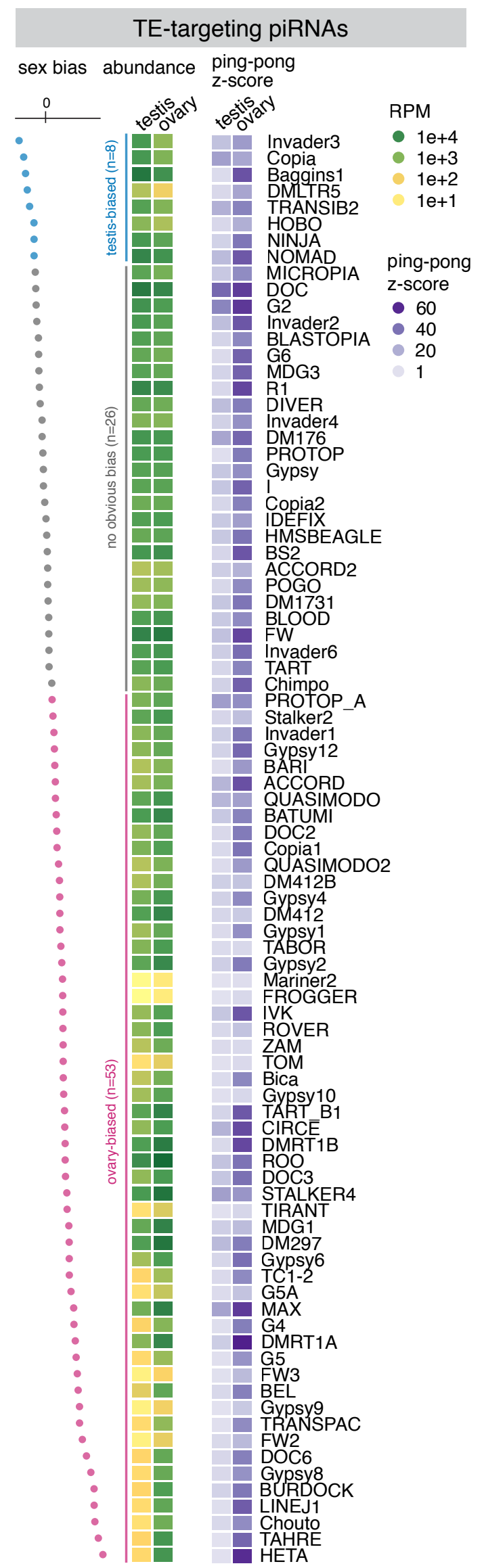

B top 10 most abundant TE-targeting piRNAs
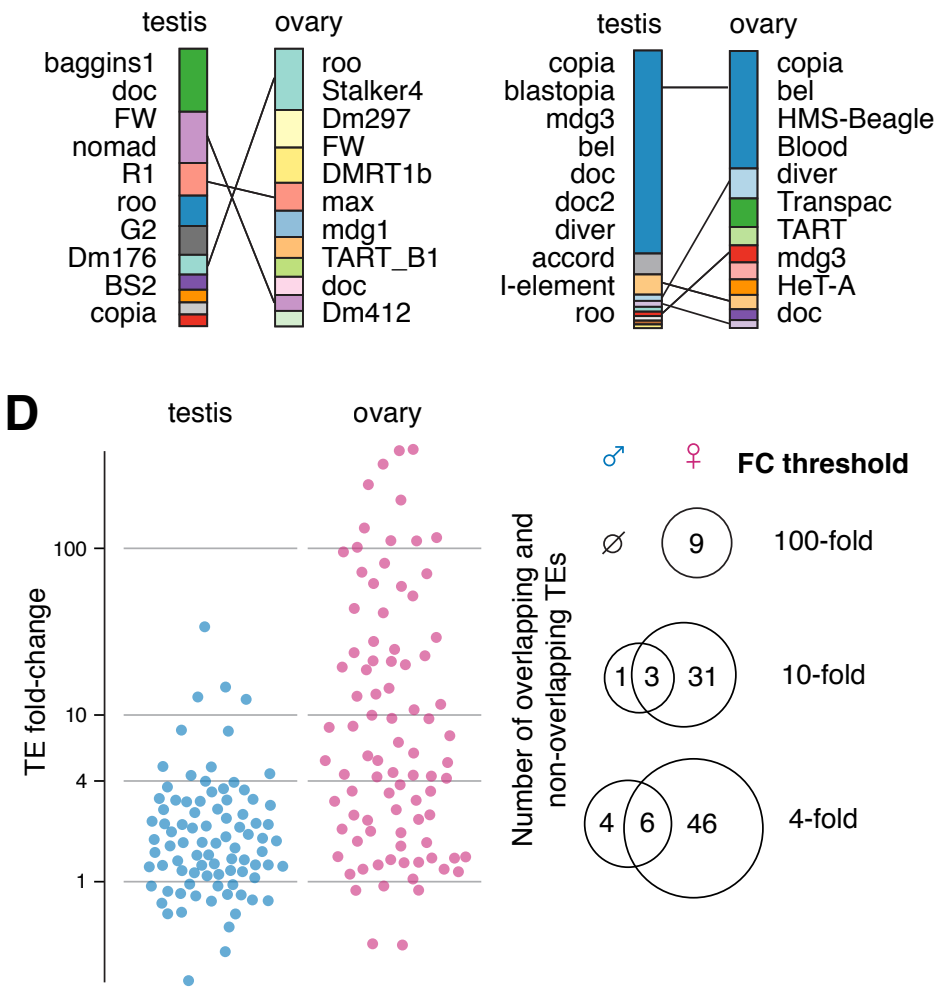

\section{E}

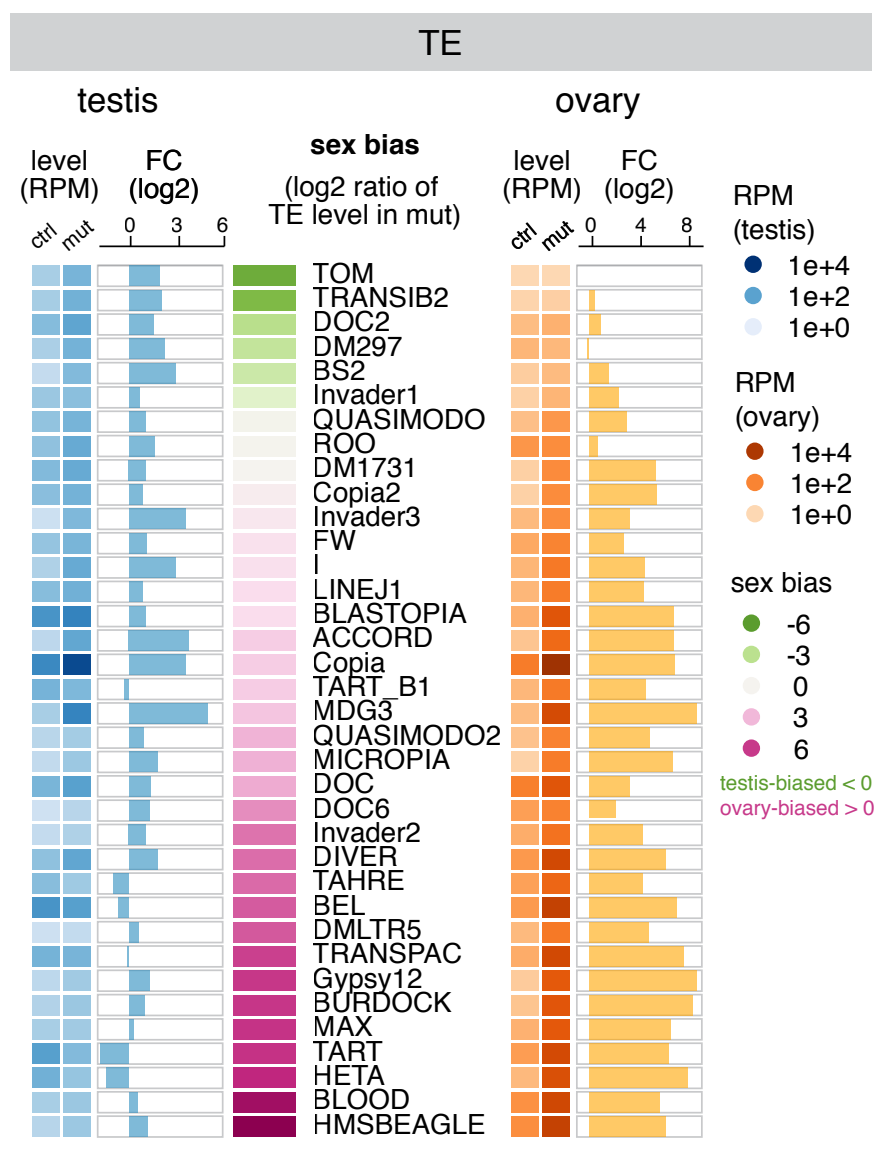




\section{Figure 3. Chen et al.}

A correlation between sex bias of TE and AS piRNA

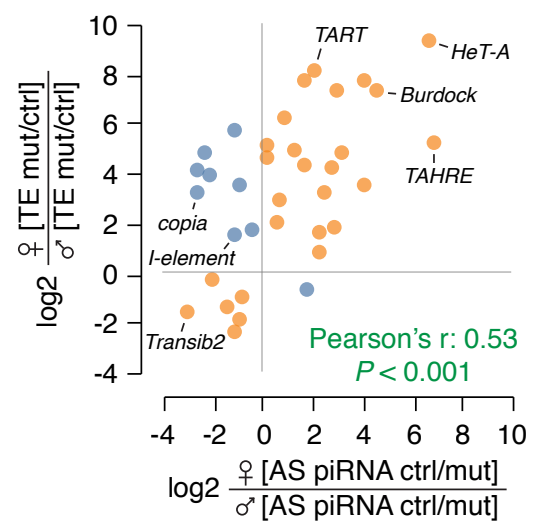

\section{C}

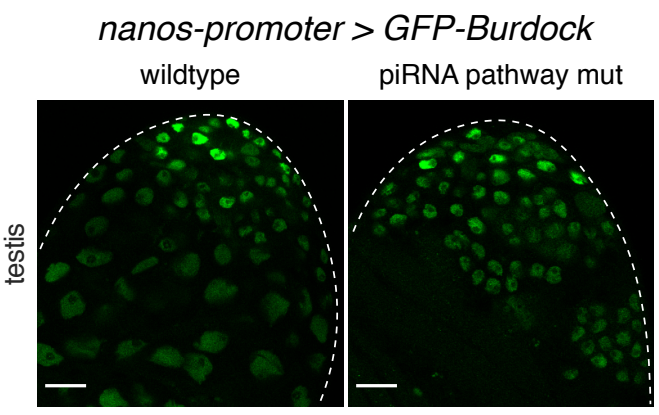

B

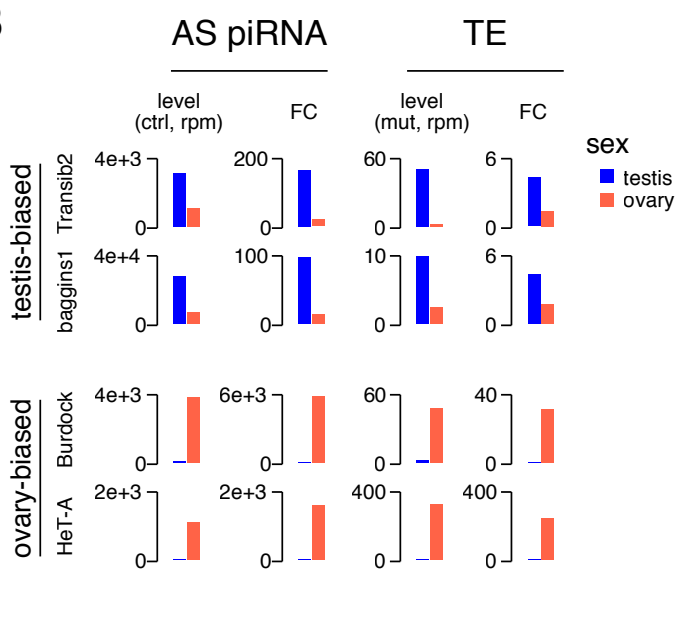

D nanos-promoter $>$ GFP-Burdock wildtype piRNA pathway mut

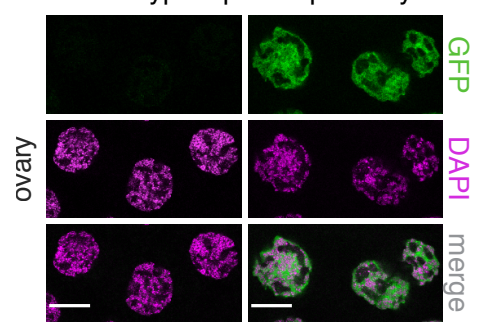


bioRxiv preprint doi: https://doi.org/10.1101/2020.08.25.266585; this version posted August 25, 2020. The copyright holder for this preprint (which was not certified by peer review) is the author/funder, who has granted bioRxiv a license to display the preprint in perpetuity. It is made available under aCC-BY-NC-ND 4.0 International license.

\section{Figure 4. Chen et al.}

A different types of piRNA reads

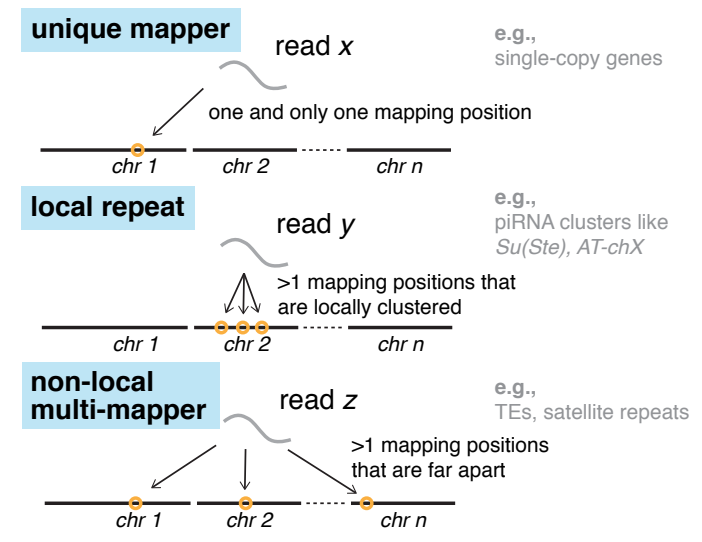

\section{C top piRNA clusters in testis}

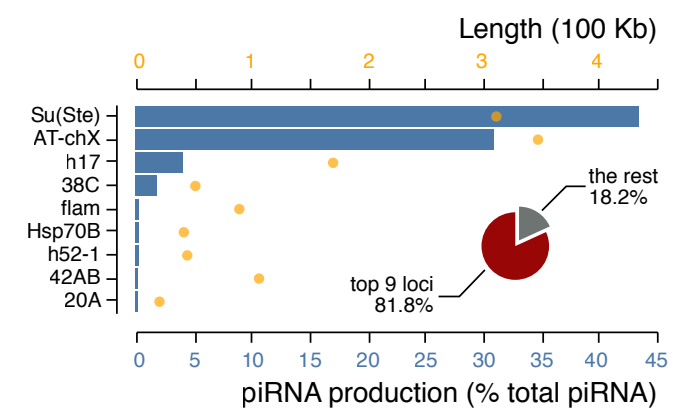

B comparison of different read-inclusion criteria on major piRNA clusters

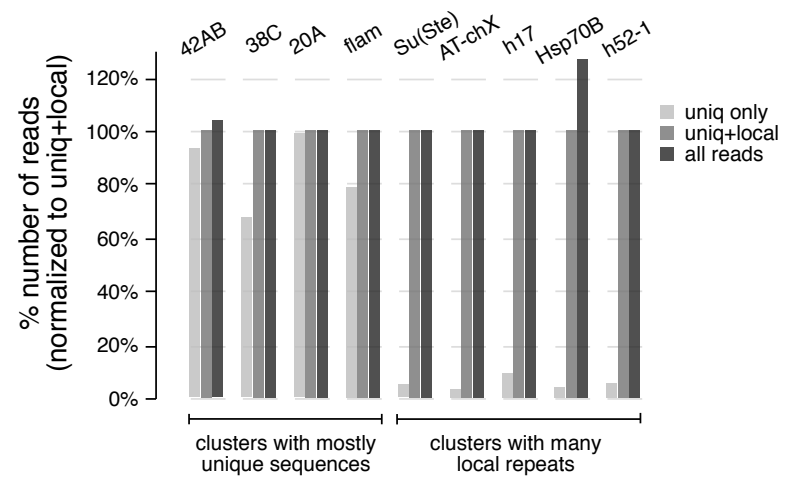

D top piRNA clusters in ovary

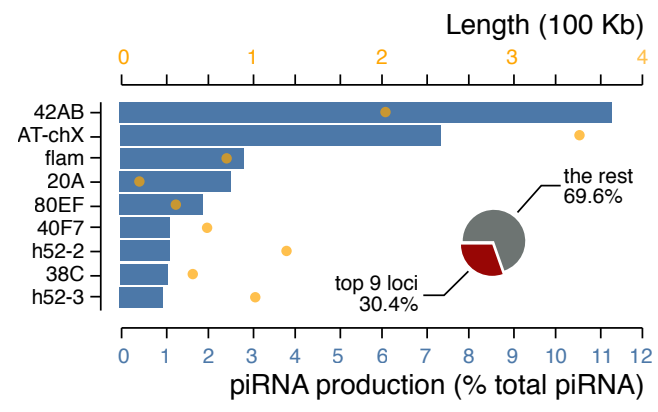

E

\begin{tabular}{rcc}
$\operatorname{chrX}(\mathrm{dm} 6)$ & $21,700,000$ & $21,900,000$ \\
\cline { 2 - 2 } clusters & flamenco & AT-chX (locally repetitive, not found outside the 20B region)
\end{tabular}

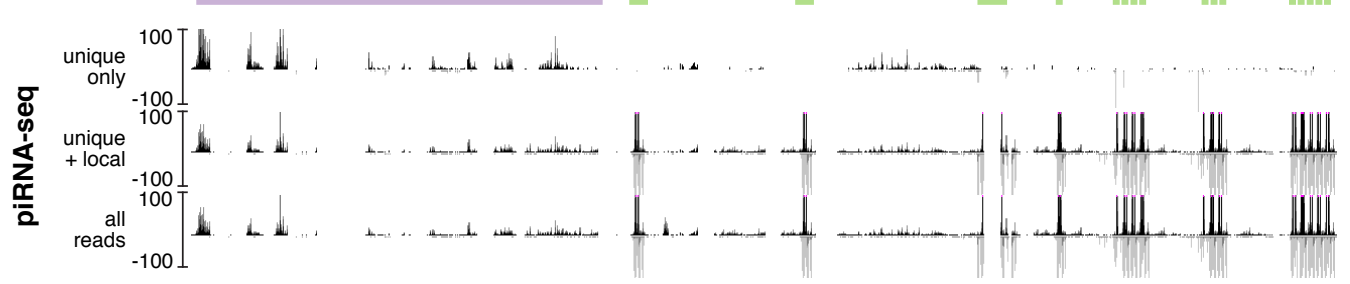

RepeatMasker

\section{$\mathbf{F}$}

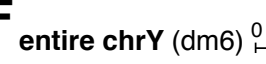

$1 \mathrm{Mb}$ $2 \mathrm{Mb}$ $3.667 \mathrm{Mb}$ piRNA clusters

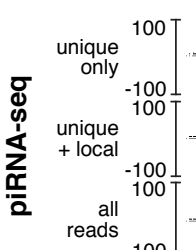

Su(Ste)-1 locally repetitive, not found outside the medial region of $Y L$

Su(Ste)-2

h17 cluster

$\begin{array}{llll}\text { all known } & \mathrm{kl}-5 \quad \mathrm{kl}-3 & \mathrm{kl}-2\end{array}$

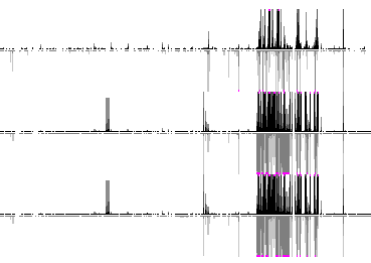

protein-encoding
genes
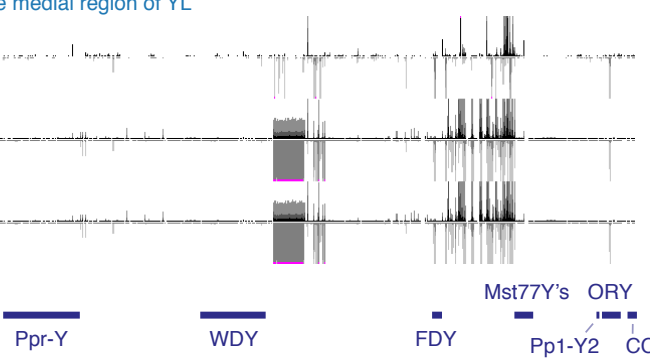


\section{Figure 5. Chen et al.}

A

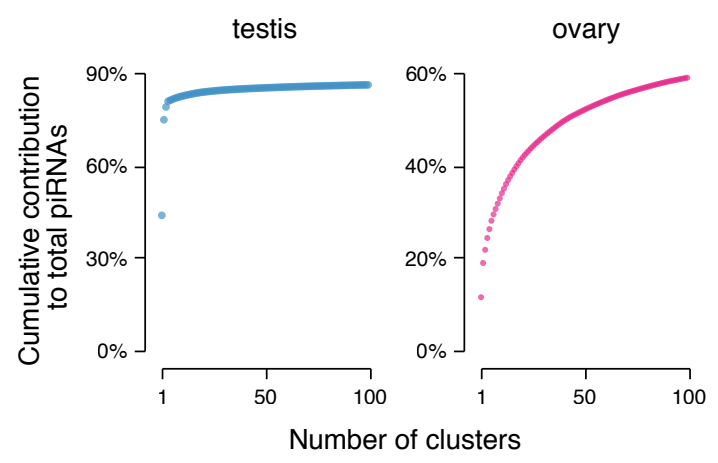

B expression of top piRNA clusters in two sexes

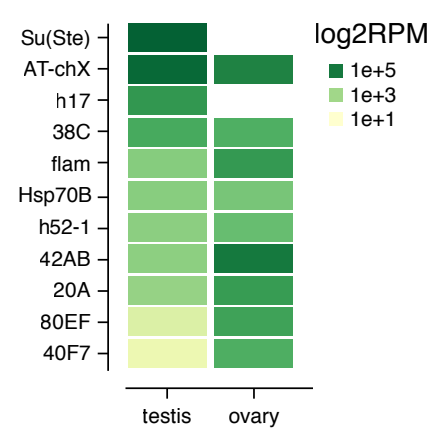

C
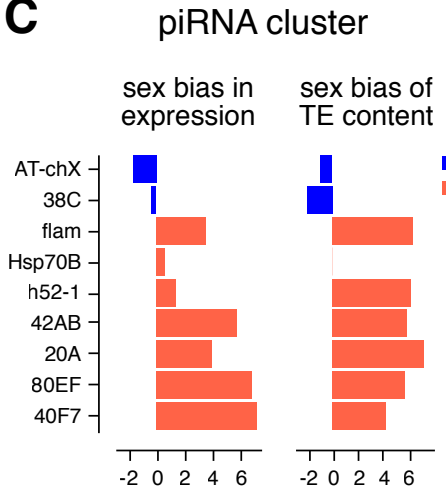

$\longrightarrow \sum x_{i} y_{i}$, where:

$x_{i}$ is the sex bias of a TE inside the cluster of interest

$y_{i}$ is the length contribution of that TE to the cluster of interest

testis-biased

ovary-biased

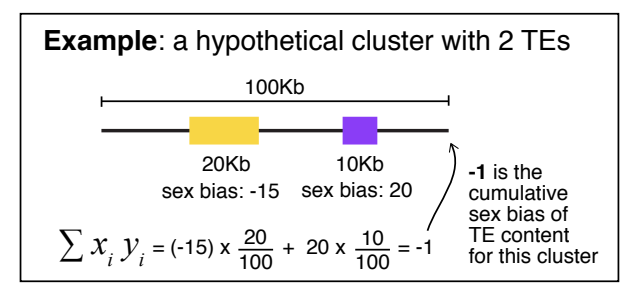

D ovary-biased TEs as top occupants of $42 A B$

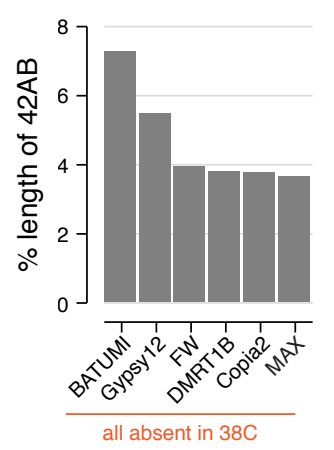

E distinct occupancy of

3 testis-biased TEs

in piRNA clusters

hobo BS2 Transib2

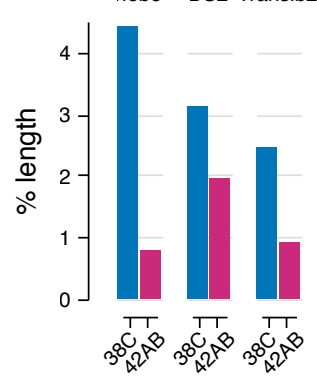

$\mathbf{F}$
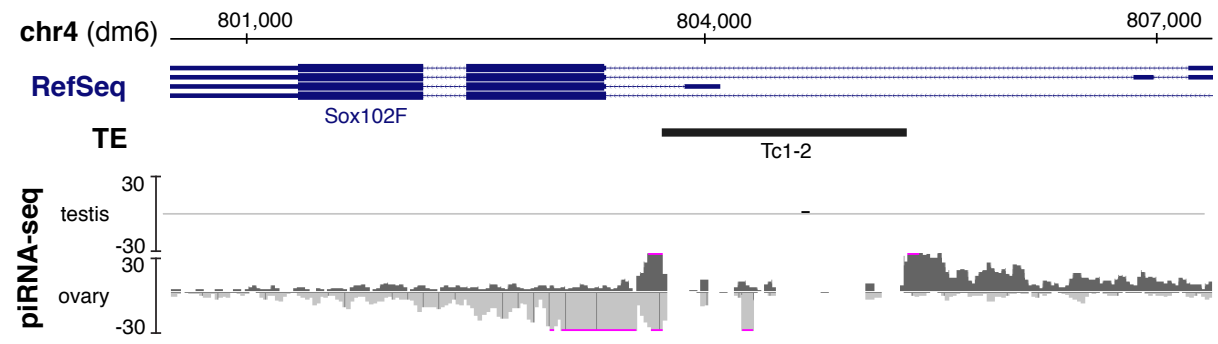
bioRxiv preprint doi: https://doi.org/10.1101/2020.08.25.266585; this version posted August 25, 2020. The copyright holder for this preprint (which was not certified by peer review) is the author/funder, who has granted bioRxiv a license to display the preprint in perpetuity. It is made available under aCC-BY-NC-ND 4.0 International license.

\section{Figure 6. Chen et al.}
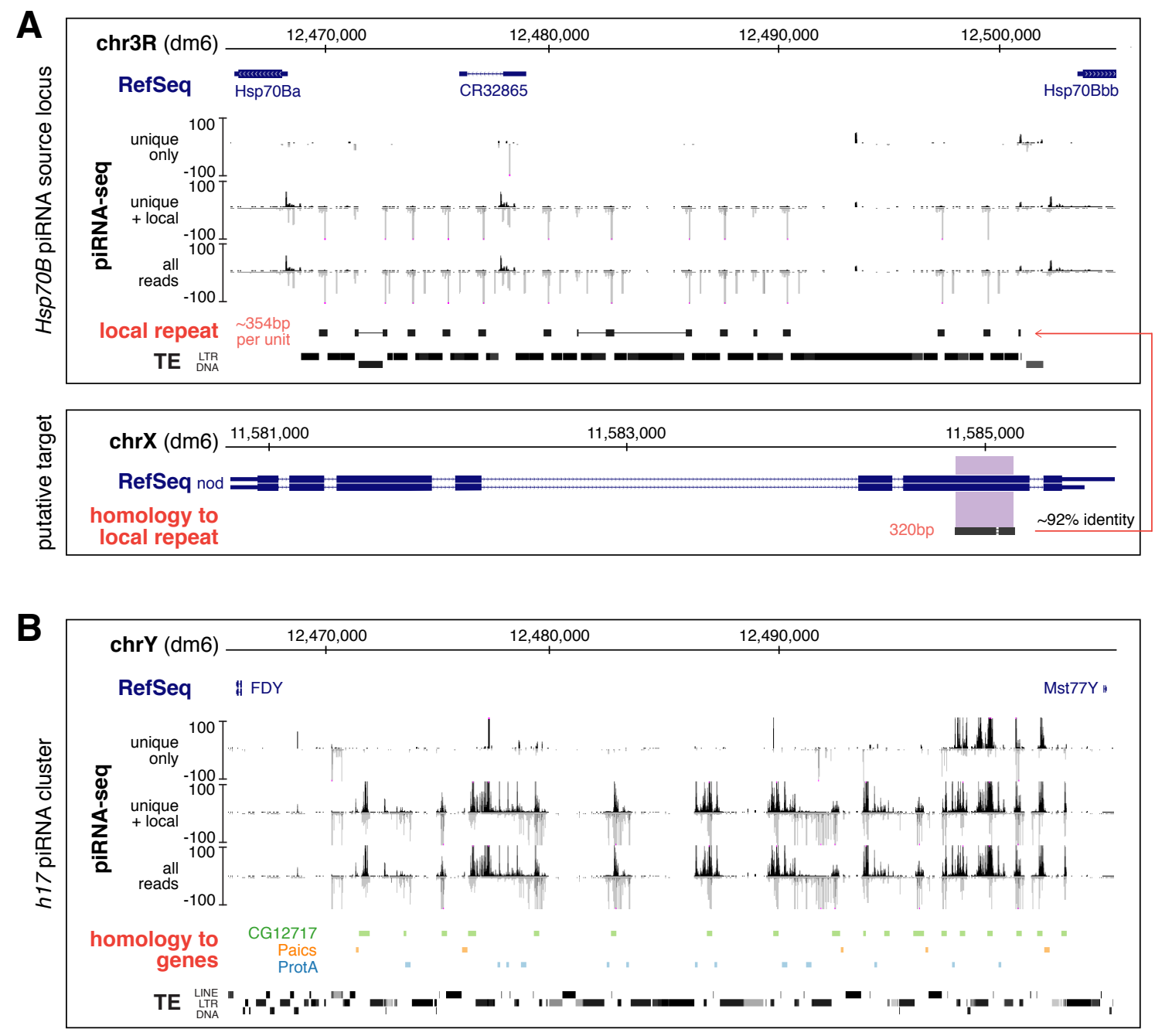

C

comparison of piRNAs mapping to four protein-encoding-genes
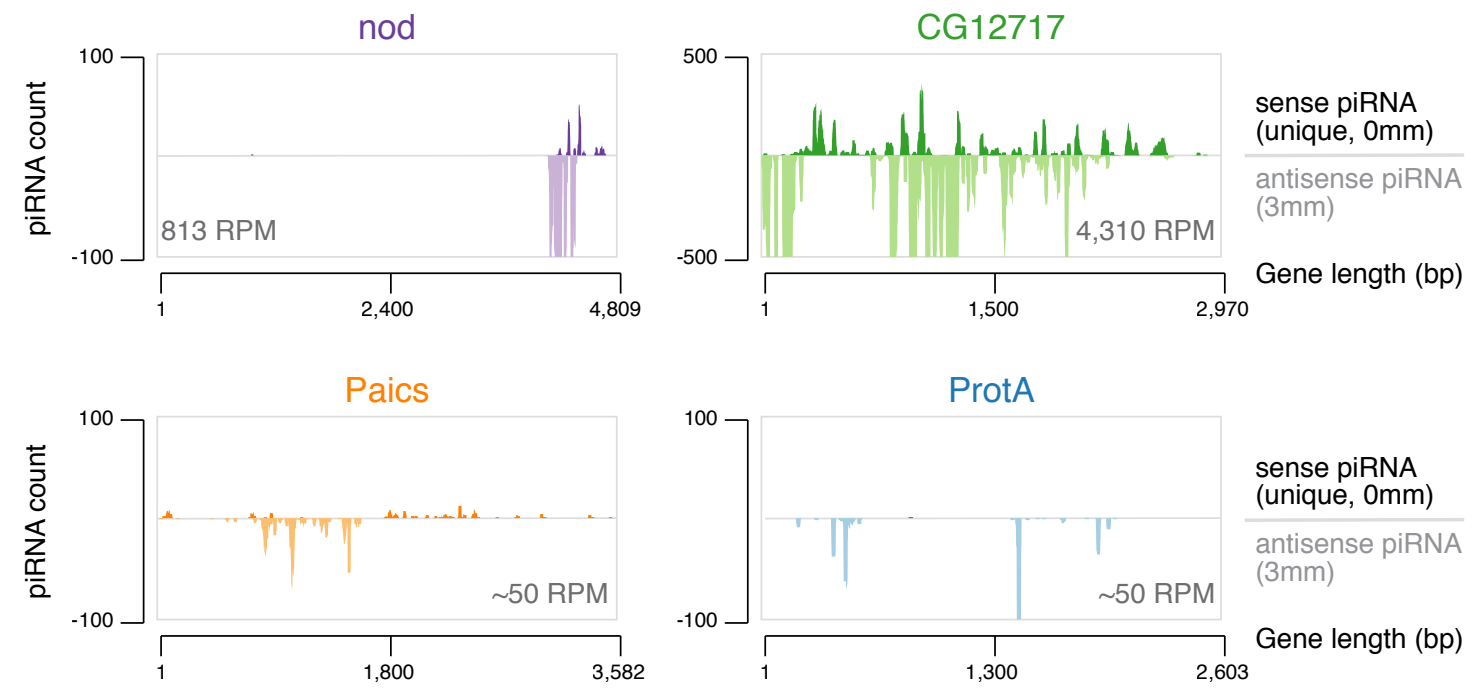


\section{Figure 7. Chen et al.}

A

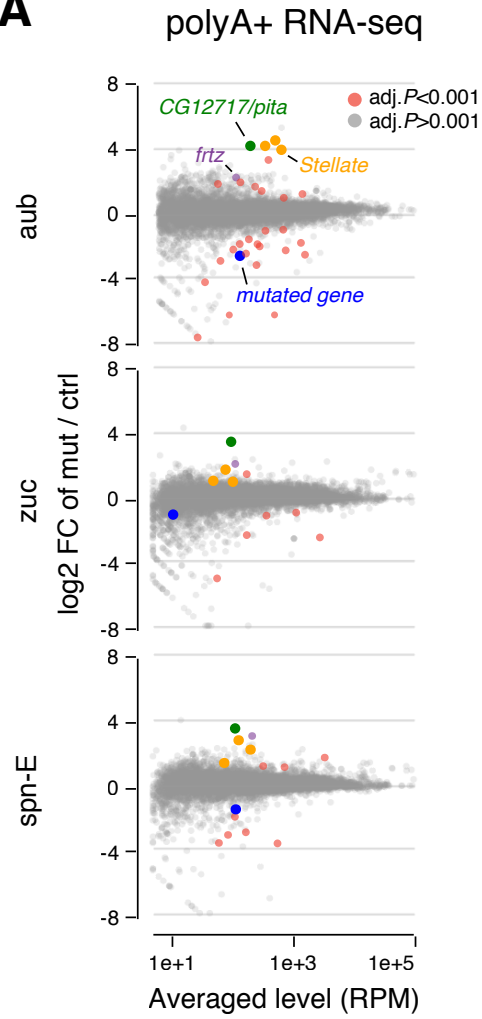

B CG12717/pita RNA in situ HCR
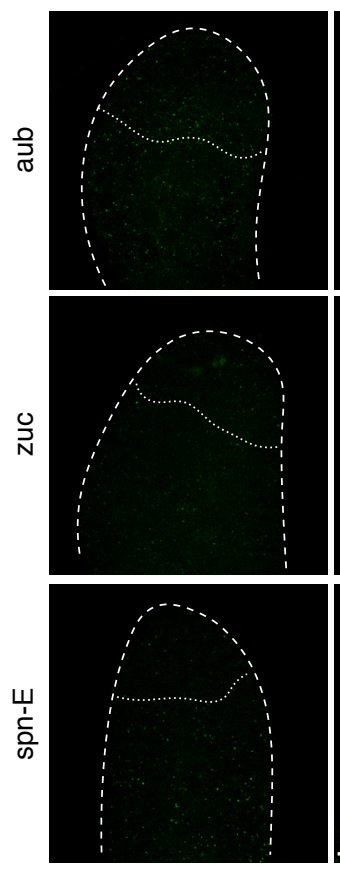

heterozygous sibling control
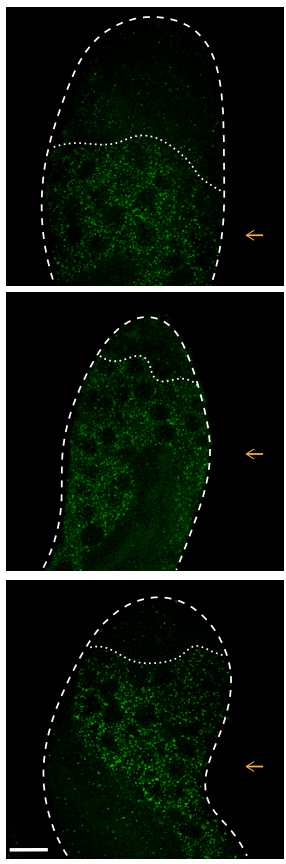

mutant

C $\begin{array}{rlll}\text { aub zuc spn-E } \\ \text { CG12717 } \\ \text { /pita } & 17.1 & 10.8 & 11.6\end{array}$

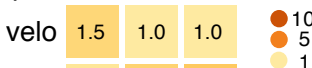
nod $1.1 \quad 1.6 \quad 1.9$

Paics $1.0 \quad 0.8 \quad 1.0$

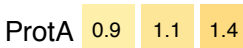

D mRNA-seq from

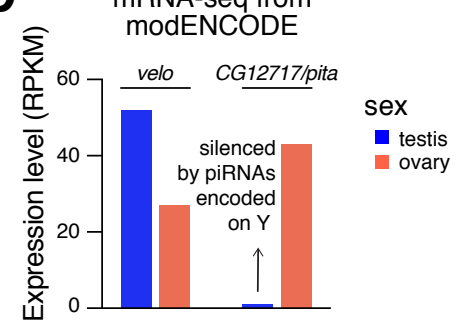

E ping-pong signature of CG12717/pita-mapping piRNAs

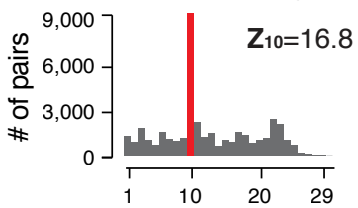

5'-5' distance of piRNA pairs (nt)

F evolution of CG12717/pita, its paralog velo, and duplication of pita

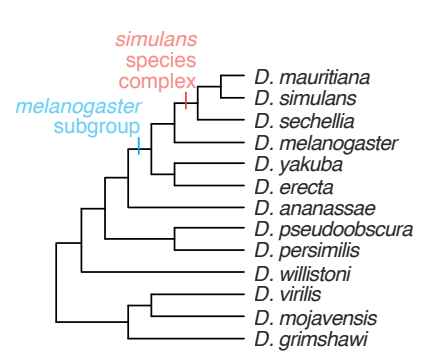

velo pita additional pita-homolog chr3L chrX heterochromatin

$$
\begin{aligned}
& \operatorname{chrX}(10), \operatorname{chr} 3 \text { (11), chrY (5), scaffold (48) } \\
& \text { scaffold (3) } \\
& \text { chrX (1), scaffold (3) } \\
& \operatorname{chrY~(20+):~h17~locus~(see~Fig.~6B)~} \\
& \begin{array}{l}
\text { present } \\
\text { absent } \\
\text { location of additional pita-homolog } \\
\text { (copy number) }
\end{array}
\end{aligned}
$$

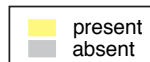

H

profiles of CG12717/pita-mapping small RNA in testis ( $\leq 3$ mismatches) size distribution D. melanogaster
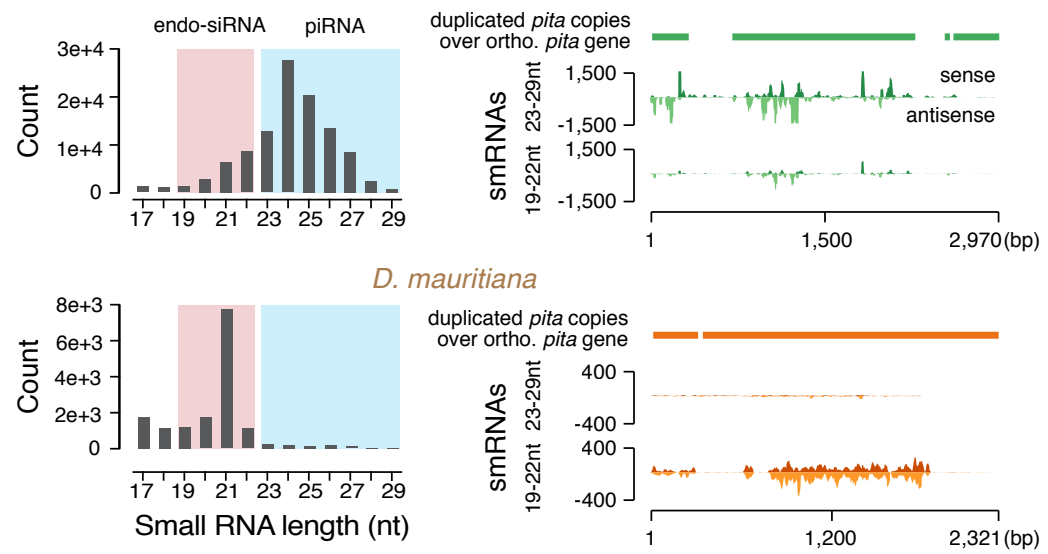

D. mauritiana

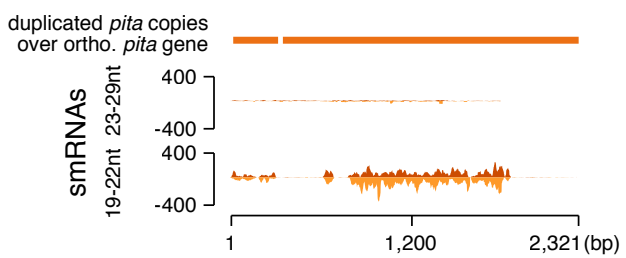

G

distribution of pita-homologous sequences in $D$. mauritiana genome ( $Y$ and unassigned scaffolds not shown)

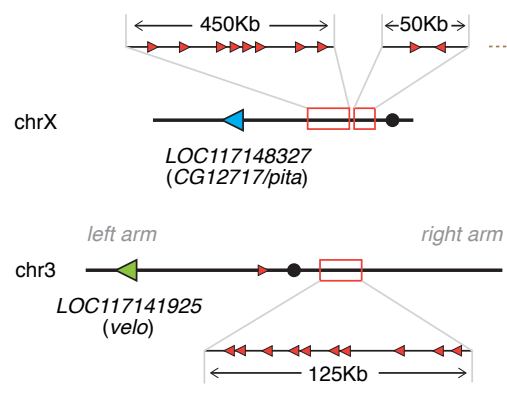

I example of large inverted repeats encompassing head-to-head pita homologs and TEs in heterochromatin (D. mauritiana)

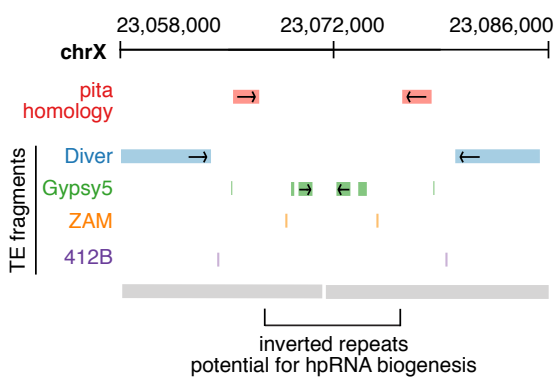


bioRxiv preprint doi: https://doi.org/10.1101/2020.08.25.266585; this version posted August 25, 2020. The copyright holder for this preprint

(which was not certified by peer review) is the author/funder, who has granted bioRxiv a license to display the preprint in perpetuity. It is made available under aCC-BY-NC-ND 4.0 International license.

\section{Figure S1. Chen et al.}

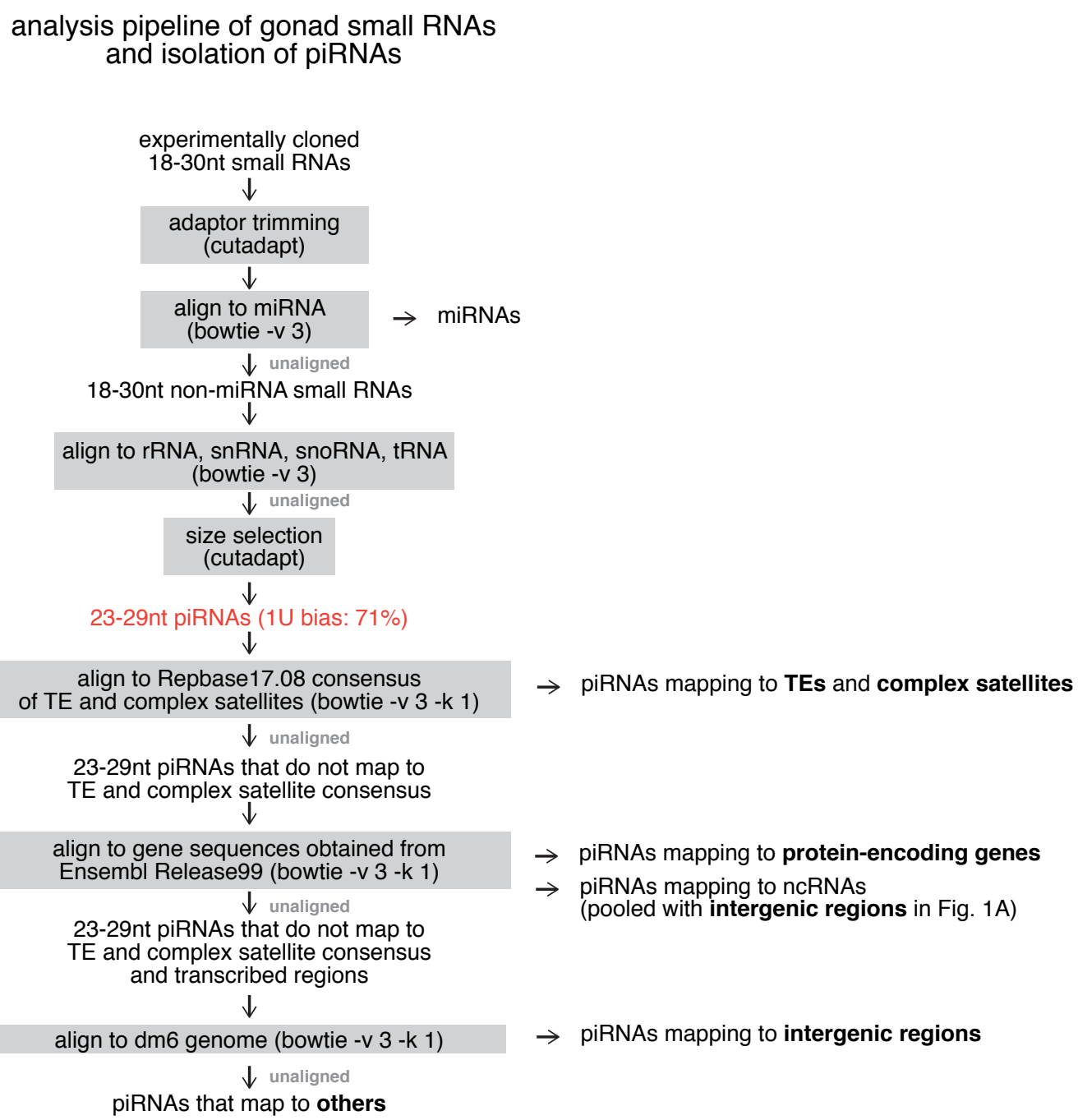


bioRxiv preprint doi: https://doi.org/10.1101/2020.08.25.266585; this version posted August 25, 2020. The copyright holder for this preprint (which was not certified by peer review) is the author/funder, who has granted bioRxiv a license to display the preprint in perpetuity. It is made available under aCC-BY-NC-ND 4.0 International license.

\section{Figure S2. Chen et al.}

A coverage of piRNA reads over complex satellite consensus
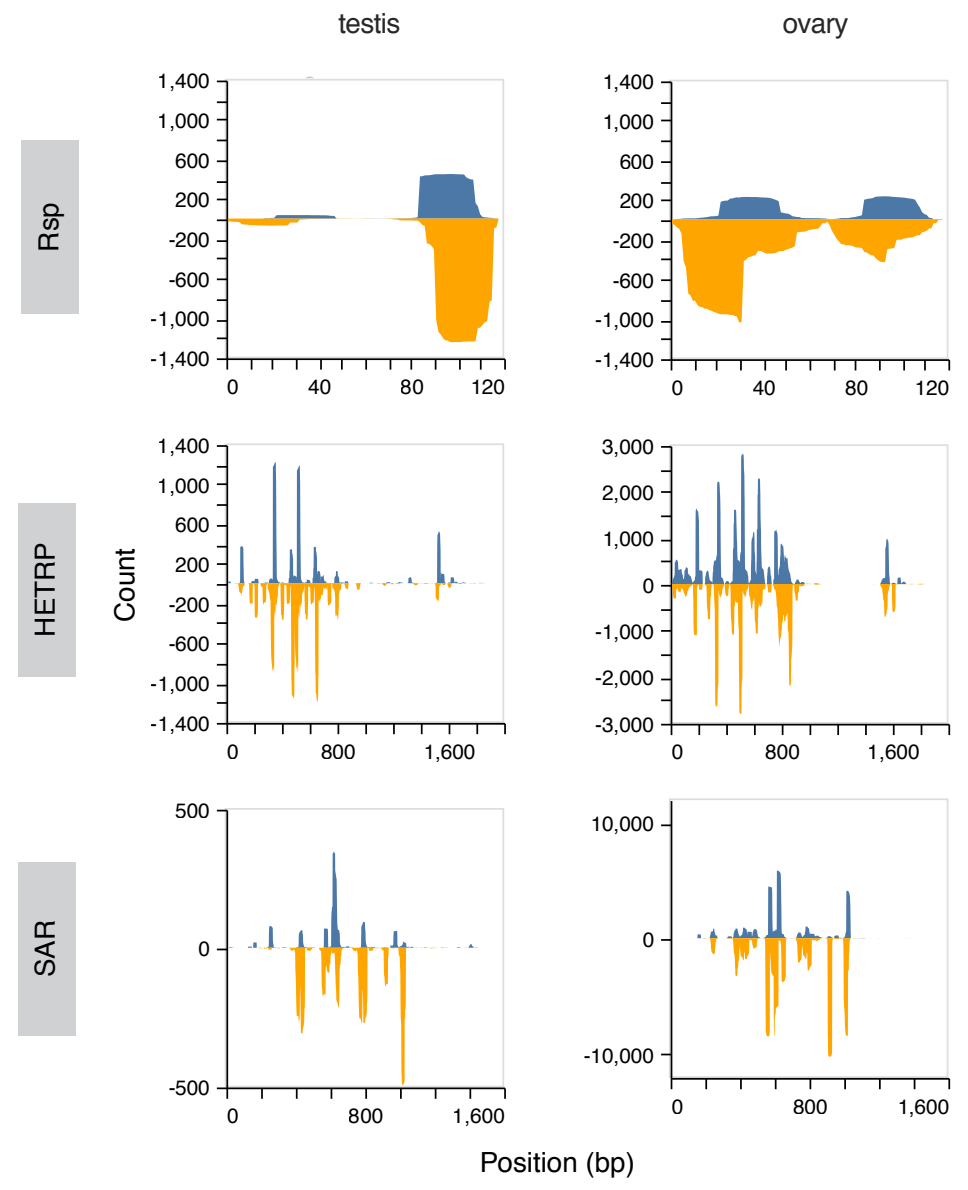

$\mathbf{B}$

complementary pairs of piRNAs

\section{Rsp consensus $118 \mathrm{bp}$}

1

CCTTCTACAGATTATTTAAACCTGGTACACAAAAACAATAAATTGACTAAGTTATGTCATTTTAGCGGTCAAATGAGTGATTTTTCGATTTCAAGTACCAGGCGAACAGAAGACACCT

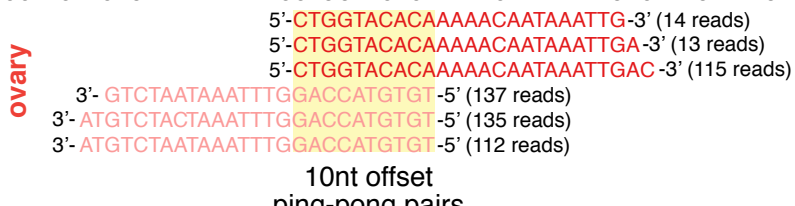

ping-pong pairs

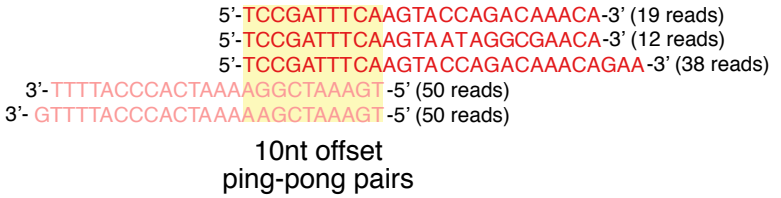

10nt offset

ping-pong pairs

'-TCCGATTTCAAGTACCAGGCGAACAG -3' (34 reads) 5'-TCCGATTTCAAGTACCAGGCGAAC -3' (144 reads) 5'-TCCGATTTCAAGTACCAGACAAAC-3' (120 reads)

3'-AGGCTAAAGTTCATGGTCT GTTT GT-5' (24 reads) near-perfect complementarity 
bioRxiv preprint doi: https://doi.org/10.1101/2020.08.25.266585; this version posted August 25, 2020. The copyright holder for this preprint (which was not certified by peer review) is the author/funder, who has granted bioRxiv a license to display the preprint in perpetuity. It is made available under aCC-BY-NC-ND 4.0 International license.

Figure S3. Chen et al.

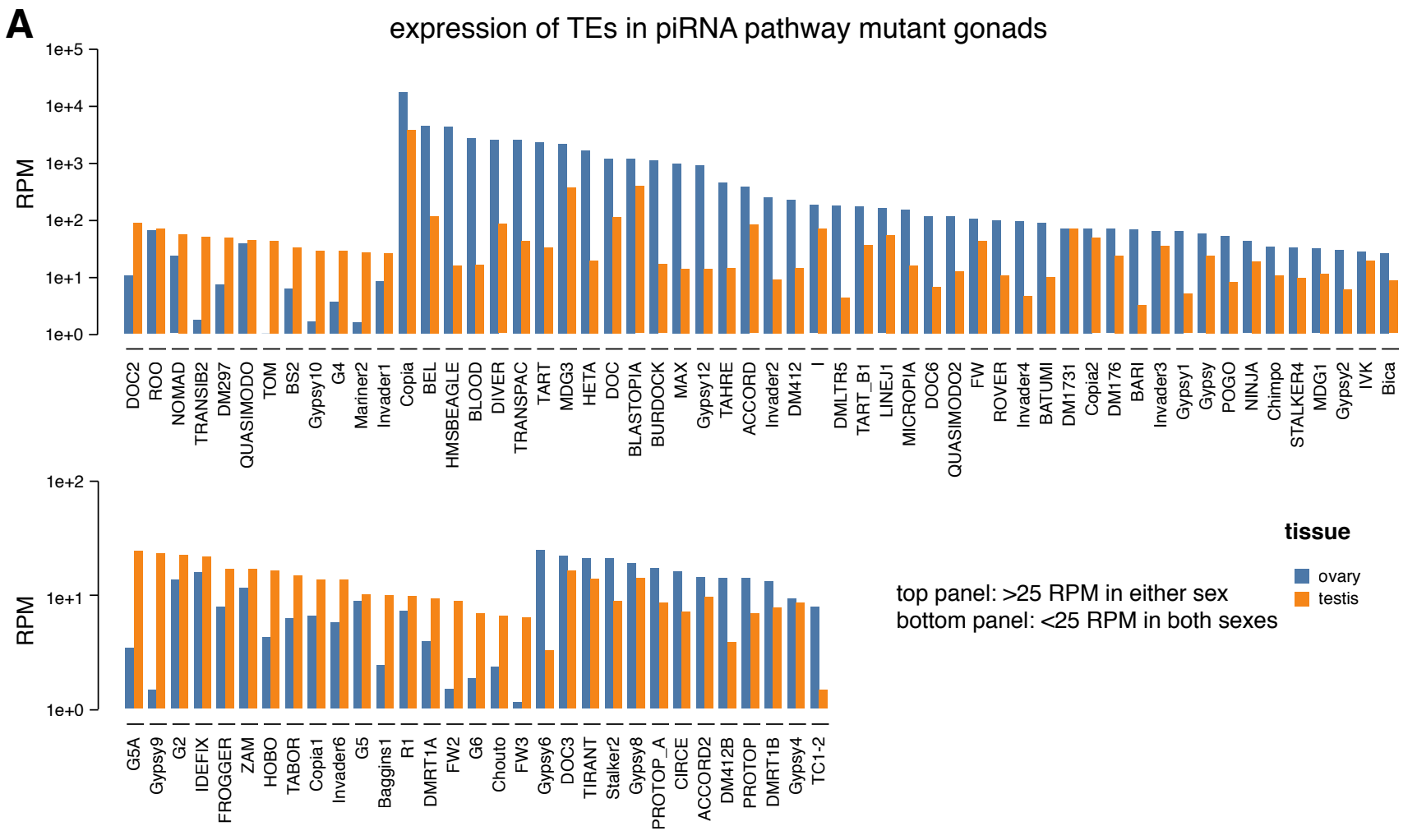

B

profiles of 36 TEs regulated by Rhi in at least one sex

\begin{tabular}{|c|c|c|c|c|c|c|c|c|c|c|c|}
\hline TE_merge & ovary_TE_fc & $\begin{array}{c}\text { ovary_silencing } \\
\text { potential }\end{array}$ & ovary_pi & ovary_pi_FC & testis_TE__f & \begin{tabular}{|c|} 
testis_silencing \\
potential
\end{tabular} & testis_pi & testis_pi_FC & Derepressed in & CAN EXPLAIN & Note \\
\hline MDG3 & 382.2 & TRUE & 2098.3 & 4.3 & 33.2 & TRUE & \begin{tabular}{|l|}
2944.7 \\
\end{tabular} & 8 & Both sex & YES & \\
\hline ACCORD & 109.6 & TRUE & 1282.2 & 15.4 & 14.4 & TRUE & 496.8 & 9.6 & Both sex & YES & \\
\hline Copia & 114.4 & TRUE & 1110.3 & 4.4 & 12.5 & TRUE & 5141.1 & 26.4 & Both sex & YES & \\
\hline Invader3 & 9.7 & TRUE & 731.9 & 20.4 & 12.1 & TRUE & 4857.3 & 81.8 & Both sex & YES & \\
\hline 1 & 22.2 & TRUE & 2632.6 & 9.3 & 7.9 & TRUE & 2586.3 & 19.6 & Both sex & YES & \\
\hline DIVER & 70.7 & TRUE & 1563.2 & 9.2 & 3.6 & TRUE & 1985.5 & 2.9 & Both sex & YES & \\
\hline MICROPIA & 100 & TRUE & 1357.9 & 25.7 & 3.5 & TRUE & 2531.6 & 124 & Both sex & YES & \\
\hline BS2 & 2.9 & TRUE & 7253.5 & 39.6 & 7.8 & TRUE & 5274.9 & 105.7 & Testis & YES & testis biased TE \\
\hline TRANSIB2 & 1.4 & TRUE & 1062.1 & 20.1 & 4.2 & TRUE & 3090.7 & 161.3 & Testis & YES & testis biased TE \\
\hline DOC2 & 1.9 & TRUE & 2090.2 & 13 & 3 & TRUE & 725.3 & 3.6 & Testis & YES & testis biased TE \\
\hline DM297 & 0.9 & FALSE & 27748.2 & 0.3 & 4.8 & FALSE & 3626.7 & 0.7 & Testis & ? & TE derepression w/o tissue-wide pirNA loss \\
\hline том & 1 & FALSE & 137.2 & 0.7 & 3.8 & FALSE & 28.7 & 1.3 & Testis & ? & TE derepression w/o tissue-wide pirNA loss \\
\hline ROO & 1.6 & FALSE & 48306.1 & 0.5 & 3.1 & FALSE & 8654.7 & 0.8 & Testis & $?$ & TE derepression w/o tissue-wide piRNA loss \\
\hline Gypsy12 & 389.1 & TRUE & 2051.2 & 29.3 & 2.5 & TRUE & 854.4 & 3.6 & Ovary & YES & ovary biased TE \\
\hline BLASTOPIA & 109.2 & TRUE & 2319.5 & 7.7 & 2.1 & TRUE & 3376 & 16.5 & Ovary & YES & ovary biased TE \\
\hline MAX & 93.7 & TRUE & 16354 & 38.9 & 1.2 & TRUE & 1505.1 & 20.5 & Ovary & YES & ovary biased TE \\
\hline Copia2 & 42.8 & TRUE & 1856.3 & 25.6 & 1.8 & TRUE & 1648.4 & 23 & Ovary & YES & ovary biased TE \\
\hline DM1731 & 40.3 & TRUE & 1047.1 & 23.9 & 2.1 & TRUE & 713.9 & 3.4 & Ovary & YES & ovary biased TE \\
\hline DMLTR5 & 27 & TRUE & 108.3 & 2.1 & 1.5 & TRUE & 377.9 & 12.8 & Ovary & YES & ovary biased TE \\
\hline Invader1 & 5.2 & TRUE & 2021.1 & 32.1 & 1.6 & TRUE & 872.7 & 41.5 & Ovary & YES & ovary biased TE \\
\hline BURDOCK & 317 & TRUE & 3755.7 & 47.1 & 2 & FALSE & 69.9 & 2 & Ovary & YES & ovary biased TE \\
\hline HETA & 238.5 & TRUE & 5725.4 & 30.9 & 0.4 & FALSE & 53.3 & 0.3 & Ovary & YES & ovary biased TE \\
\hline TRANSPAC & 192.6 & TRUE & 751.1 & 6.3 & 0.9 & FALSE & 43.6 & 2 & Ovary & YES & ovary biased TE \\
\hline BEL & 130.6 & TRUE & 2245.7 & 8.7 & 0.6 & FALSE & 145.4 & 0.5 & Ovary & YES & ovary biased TE \\
\hline TART & 80.1 & TRUE & 4354.2 & 2.6 & 0.3 & FALSE & 2756.7 & 0.6 & Ovary & YES & ovary biased TE \\
\hline HMSBEAGLE & 69.2 & TRUE & 2461 & 4.2 & 2.3 & FALSE & 1871.8 & 1.7 & Ovary & YES & ovary biased TE \\
\hline TART_B1 & 23.2 & TRUE & 14384.2 & 15 & 0.8 & FALSE & 2709.2 & 1.7 & Ovary & YES & ovary biased TE \\
\hline LINEJ1 & 20.5 & TRUE & 2184.4 & 17 & 1.9 & FALSE & 40.4 & 1 & Ovary & YES & ovary biased TE \\
\hline Invader2 & 19.6 & TRUE & 2859.8 & 7.1 & 2.1 & FALSE & 4728.2 & 1.2 & Ovary & YES & ovary biased TE \\
\hline TAHRE & 18.9 & TRUE & 5589 & 53.1 & 0.5 & FALSE & 74.4 & 0.5 & Ovary & YES & ovary biased TE \\
\hline DOC & 9.3 & TRUE & 12492.9 & 14.8 & 2.6 & FALSE & 22434.2 & 2 & Ovary & YES & ovary biased TE \\
\hline QUASIMODO & 8.2 & TRUE & 5956.2 & 2.2 & 2.1 & FALSE & 2290 & 1.4 & Ovary & YES & ovary biased TE \\
\hline $\mathrm{FW}$ & 6.7 & TRUE & 22035 & 6.5 & 2.2 & FALSE & 14250.2 & 1.4 & Ovary & YES & ovary biased TE \\
\hline DOC6 & 4.4 & TRUE & 2051.2 & 9.3 & 2.5 & FALSE & 69.3 & 1.8 & Ovary & YES & ovary biased TE \\
\hline QUASIMODO2 & 28.6 & FALSE & 1169.4 & 1.3 & 1.9 & TRUE & 351.7 & 5.4 & Ovary & ? & TE derepression w/o tissue-wide piRNA loss \\
\hline BLOOD & 50.9 & FALSE & 5419.9 & 1 & 1.5 & FALSE & 3524.8 & 0.9 & Ovary & ? & TE derepression w/o tissue-wide piRNA loss \\
\hline
\end{tabular}


bioRxiv preprint doi: https://doi.org/10.1101/2020.08.25.266585; this version posted August 25, 2020. The copyright holder for this preprint (which was not certified by peer review) is the author/funder, who has granted bioRxiv a license to display the preprint in perpetuity. It is made available under aCC-BY-NC-ND 4.0 International license.

\section{Figure S4. Chen et al.}

$\mathbf{A}$

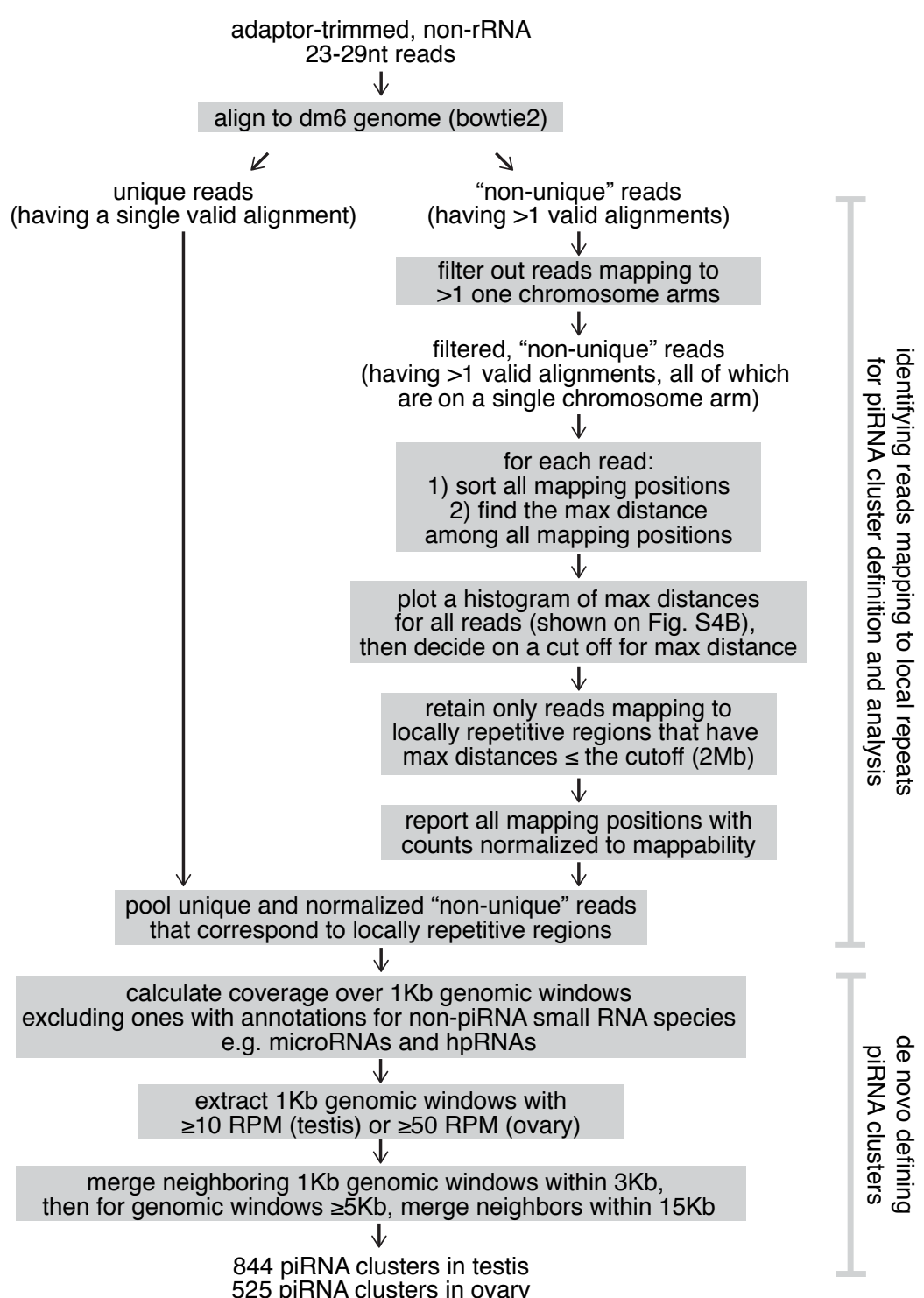

B

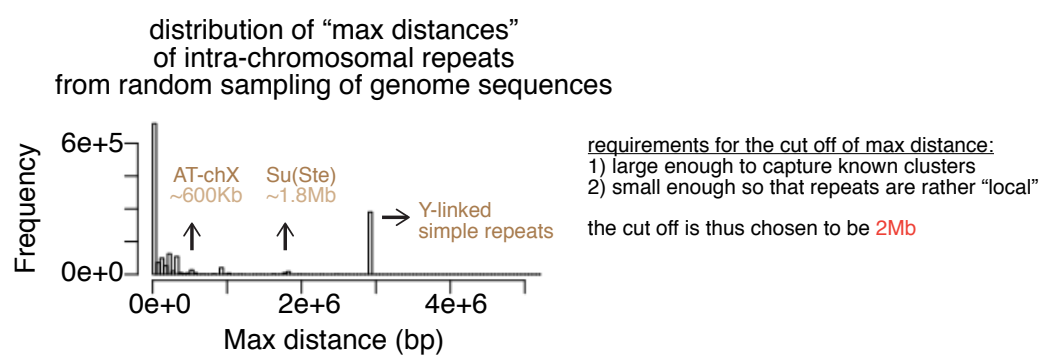


bioRxiv preprint doi: https://doi.org/10.1101/2020.08.25.266585; this version posted August 25, 2020. The copyright holder for this preprint (which was not certified by peer review) is the author/funder, who has granted bioRxiv a license to display the preprint in perpetuity. It is made available under aCC-BY-NC-ND 4.0 International license.

\section{Figure S5. Chen et al.}

A

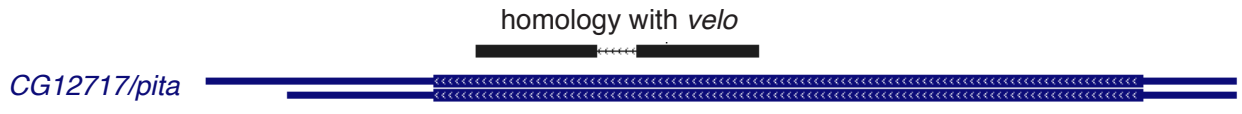

B

alignment of CG12717/pita-related fragments at $h 17$ locus along the CG12717/pita gene

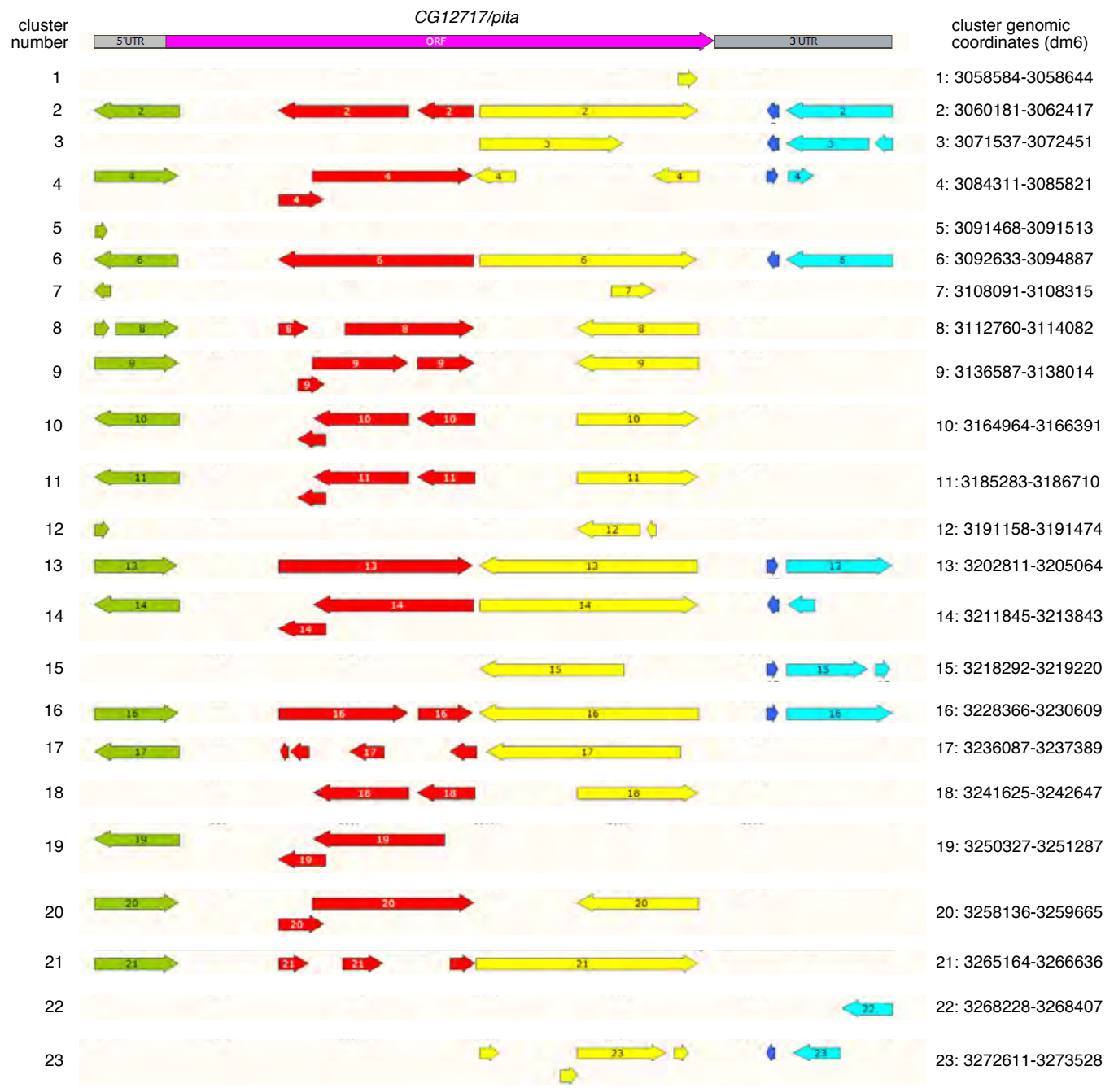

